\title{
An investigation of the flow characteristics in the bootdeck region of a scale model notchback saloon vehicle.
}

\author{
N J Lawson, N Faucompret, K P Garry \\ Aerodynamics Group \\ Department of Aerospace Sciences, \\ Cranfield University, Cranfield, \\ Bedfordshire, MK43 0AL. \\ Tel: $01234754619 \quad$ Fax: 01234758207 \\ Email:n.lawson@cranfield.ac.uk
}

\begin{abstract}
The results of an experimental investigation of the bootdeck flow structure of $36 \%$ scale model notchback car are presented together with a general review of advanced laser diagnostic techniques suitable for large scale wind tunnel flow measurement. The tests were used to characterise the flow behaviour over the Reynolds number range $0.74 \times 10^{6}$ to $4.93 \times 10^{6}$ in the Cranfield University $2.4 \mathrm{~m} \times 1.8 \mathrm{~m}$ wind tunnel. The experiments involved flow visualisation, rear bootdeck and backlight 3D stereoscopic PIV measurements and mean static and unsteady static pressure measurements. Initial results from the flow visualisation suggested flow asymmetries originating in the backlight region which were sensitive to Reynolds number. The PIV data and static pressure data, however, found little or no sensitivity of the flow to Reynolds number with consistent flow structure and levels of unsteadiness from the backlight to the rear bootdeck region. At this stage no definitive reasons can be given for the discrepancies between the flow visualisation and the other data although the flow visualisation data was particularly difficult to interpret near the backlight and the near wake structure may be bi-stable in this Reynolds number range. More detailed 3D stereoscopic, time-resolved PIV flow data are now planned to conclusively quantify the correct flow structure and it's sensitivities.
\end{abstract}

Keywords: PIV, automotive wakes, pressure measurement, flow visualisation

\section{NOTATION}

$\begin{array}{ll}R & \text { reattachment point } \\ \infty & \text { freestream }\end{array}$

\section{Abbreviations}

$\begin{array}{ll}\mathrm{C}_{\mathrm{p}} & \text { pressure coefficent } \\ \mathrm{C}_{\mathrm{pf}} & \text { corrected pressure coefficient } \\ f & \text { shedding frequency } \\ \mathrm{L} & \text { car length } \\ \mathrm{PIV} & \text { particle image velocimetry } \\ \mathrm{Re} & \text { Reynolds number } \\ \mathrm{St} & \text { Strouhal number (based on reattachment length } \mathrm{X}_{\mathrm{R}} \text { ) } \\ \mathrm{U}_{\infty} & \text { Freestream velocity } \\ \mathrm{X}, \mathrm{y}, \mathrm{z} & \text { crosswind, vertical, freestream axes } \\ \mathrm{U}, \mathrm{V}, \mathrm{w} & \mathrm{x}, \mathrm{y}, \mathrm{z} \text { velocity components } \\ \mathrm{W} & \text { car width } \\ \mathrm{X}_{\mathrm{R}} & \text { reattachment length } \\ \mathrm{N} & \text { number of correlation particle image pairs }\end{array}$




\section{INTRODUCTION}

Flows in the rear region of automotive vehicles are particularly complex and often unsteady in nature. This results in significant challenges both in the measurement of the flows experimentally and the modeling of the flows numerically [1].

The most basic flow structure has been investigated by Carr [2] who demonstrated the existence of a transverse vortex behind the backlight of a notchback vehicle. More recently, using model testing and numerical modelling at low Reynolds number, Nouzawa et. al. [3] established an alternative structure of the near-wake. Here the vortex behind the backlight window consisted of an arch shape, but with the same characteristics as the transverse vortex proposed by Carr [2], but to which two trailing vortices are added originating from the rear-pillar flow separation. Further numerical developments at low Reynolds number have shown that the arch vortex exhibits a characteristic frequency consistent with a shedding frequency scale to the vehicle characteristic length and freestream velocity. Further work by Jenkins [4] has shown that the flow separation occurring at the roof trailing edge was dominated by two bootdeck vortices, contra rotating and in an opposite sense to the rear-pillar vortices, formed behind the backlight and extended toward the centre of the bootdeck. However, the investigation did not allow a complete description of the flow structure.

Work by Carr [5] has also proposed, that that three basic flow structures exist in the bookdeck region which depend on the geometric shape of the vehicle described in terms of the backlight angle $\alpha$, the declination angle $\beta$ and the bootdeck length $L$. These three flow configuration were linked to generic vehicle types described as the Notchback, the Estate and the Fastback car [5]. Of these three types, published work is most detailed for the Notchback and Estate car configurations.

For the Notchback car, recent work by Gilhome [6,7] has focused on an experimental investigation of the flow structure over a sedan vehicle. The work has combined on-road and wind tunnel full-scale tests at Reynolds numbers up to $6.5 \times 10^{6}$. Both the time-averaged and instantaneous near-wake structure were analysed. Surface flow visualisation also indicated the presence of unstable node points (or nodes of attachment), saddle points (a singularity between inward and outward flows) and unstable foci centres consistent with a vortex stretching from those points in the streamwise direction. Work by Jenkins [4] on an Estate type configuration also confirmed the existence of similar flow structures. These basic flow structures are summarised in Figure 1.

Investigation of the unsteady nature of the flow field in this region leads to a dominant unsteady horseshoe vortex structure which is periodically shed from the rear-pillars. This periodic shedding results in the separation region behind the backlight also having unstable characteristics. Gilhome's analysis [6] of unsteady surface pressures and corresponding spectra revealed two characteristic frequencies associated with the near-wake of the vehicle. The corresponding Strouhal numbers, based on the reattachment length, were 0.11 and 0.42 respectively where Strouhal number is defined by:

$$
S t=\frac{f x_{R}}{U_{\infty}}
$$

given a vortex shedding frequency $f$, a reattachment length $x_{R}$ and a freestream velocity of $U_{\infty}$. The lower frequency was associated with a flapping motion of the shear layer, whereas the higher frequency was attributed to the vortex shedding from the rear-pillar.

In the present study, data obtained using a nominally $1 / 3^{\text {rd }}$ scale passenger saloon car model is presented using conventional time-averaged pressure measurement, unsteady pressure measurement, flow visualisation and the advanced optical measurement technique particle image velocimetry (PIV) $[8,9]$. Given the growing use of these advanced optical techniques, a number of optical methods which are available to the wind tunnel instrumentation engineer, are also reviewed. This is considered important, as the availability of measurement methods such as PIV now offer the potential to increase understanding of both the time-averaged and unsteady flow fields. To this end, results from the measurements in this paper confirm a number of the complex flow structures predicted by previous workers and also show unexpected sensitivity of the flow to Reynolds number which has not been reported before. 


\section{ADVANCED OPTICAL METHODS}

A number of advanced optical techniques are available for wind tunnel measurement. Although these methods are generally highly mature, application of these techniques to automotive wind tunnels, which are typically large scale and are low to medium speed, presents significant challenges. These challenges include optical access, the large volumes to be measured and ensuring sufficient seeding particles of the right size are present in the flow region of interest. In this section, we will briefly review three of the techniques available for wind tunnel measurement including laser Doppler anemometry (LDA), particle image velocimetry (PIV) and Doppler global velocimetry (DGV).

\subsection{Laser Doppler Anemometry (LDA)}

LDA is a well established point wise velocity measurement technique. Its maturity is reflected in detailed descriptions of the technique as published by Drain [10], Durst et al [11] and more recently Albrecht et al [12]. In another configuration similar to LDA, phase Doppler anemometry (PDA) is used to measure both velocity and particle size simultaneously [13]. The most common 3D LDA set-up is illustrated in Figure 2. In this configuration, three colours are used in crossed beam arrangements to interrogate a single coincident point in the fluid and the three individual components are then transformed into 3D velocity data. Many of the commercial systems combine the transmission and receiving optics for one or two components into one probe in a backscatter arrangement. This has an advantage when optical access may be limited to a single window. LDA offers high accuracy and spatial resolution (typically less than $0.5 \%$ error in mean velocity) and allows measurement of fundamental parameters such as turbulence intensity and Reynolds stresses. But as instantaneous flow structure cannot be measured, LDA measurements are sometimes complemented by PIV flow studies as well.

Given the maturity of LDA as a turn-key technique and the limited review space allowed in this article, only a small number of application examples can be listed here. In work by Jeffrey and Zhang [14], 3D LDA measurements were taken from a Gurney flap on the trailing edge of a NACA 0012 symmetrical aerofoil section. Measurements were completed in both a $3.5 \mathrm{~m} \times 2.5 \mathrm{~m}$ and $2.1 \mathrm{~m} \times 1.7 \mathrm{~m}$ wind tunnel working sections. In this case specialised stable probe mounts and a commercial smoke generator were used. Another example of application of LDA in large scale facilities includes helicopter rotor model measurements [15]. In both cases the optics set-up required careful attention with large focal length lenses at significant cost in order to obtain the required accuracy and performance.

\subsection{Particle Image Velocimetry (PIV)}

PIV is a development of the laser speckle velocimetry (LSV) technique as originally demonstrated by Dudderar et. al. in 1977 [16]. PIV is a planar velocity measurement technique which in its simplest form uses a multiple pulsed laser sheet placed in the plane of interest of the seeded fluid (see Figure 3). Images are recorded from the light sheet using either a digital or a photographic camera and subsequently processed using optical or digital correlation techniques to yield a twodimensional (2D) velocity vector map of the flow $[8,9]$.

More recent advances allow the simpler 2D PIV technique to be extended to 3D by using stereoscopic methods [17] and the commercial turn-key systems permit relatively simple application of stereoscopic PIV to an area of interest. Details of such a system are outlined later in this paper as the technique is applied to our wind tunnel model. Further advances in the 3D PIV technique to a 3D volume measurement also include scanning sheet methods [18], defocus methods [19] and holographic methods [20,21], although none of these as yet have been comprehensively applied to a large wind tunnel environment. In addition, high speed digital cameras now allow measurement of PIV data, in 2D and stereoscopic arrangements, at $\mathrm{kHz}$ frame rates thus allowing the study of temporal complex turbulent flow structures [22,23]. A comprehensive guide outlining the major developments and methods in PIV over the last 10 years has been published by Raffel et al [24]. 
Application of the PIV method to large wind tunnels has been successful in a number of cases. These include PIV wake measurements from helicopter rotors and wings in facilities with working sections areas as large as $9.5 \mathrm{~m} \times 9.5 \mathrm{~m}[25,26]$. The size of the facilities has required special systems to be developed such as large scale atomisers for seeding, customised light sheet optics and specialised camera mounts. Also 2D PIV measurements of a wing tip vortex from a NACA 0015 profile have been taken using another large scale facility $(9 \mathrm{~m} \times 9 \mathrm{~m}$ working section area) with successful tracking of vortex flow structures [27]. Other work includes wake measurements from a rotor system [28] and wakes generated behind an Ahmed geometry generic car model [29]. In the later case, the PIV data has shown the vortex drag from the Ahmed body to peak at a hatchback angle of $30^{\circ}$.

\subsection{Doppler Global Velocimetry (DGV)}

DGV, like PIV, is a planar technique which will measure up to three velocity components from the flow. The technique was first conceived by a number of independent research groups over 10 years ago [30,31]. Comprehensive reviews on DGV have been completed by a number of authors [32-36]. Described briefly and with reference to Figure 4 which shows a 1D system, the technique directly measures the Doppler shift of light scattered from seeding particles in a light sheet given the wavelength of the incident light and knowing the geometry of the configuration which can also be obtained through direct calibration methods.

In practice this Doppler measurement is achieved by the use of an iodine absorption filter that is integrated into the imaging optics and a frequency stabilised laser tuned to the required point on the iodine absorption line. At each corresponding point in the flow, the resultant signal image will then have pixel values proportional to the seeding particles velocity with the geometry of the setup referenced to a sensitivity vector and the laser frequency. Thus the technique offers the potential to yield one velocity vector from each pixel and with optical processing speeds, at near CCD frame rates. Also since the technique is simply dependent on the Doppler shift, many of the seeding restrictions of PIV can be removed by using smaller seeding with corresponding gains in scattering efficiency [8] and advantages in large scale wind tunnel environments. Disadvantages, however, as with LDA, include the requirement for three views or different laser sheet directions [37] to obtain the three components. Also the accuracy of measurement is dependent on laser and iodine cell stability [38] and sensitivity vector error at each pixel and this restricts the best expected errors to between $0.5-1 \mathrm{~m} / \mathrm{s}[37,39,40]$. Thus the technique is generally unsuitable for low speed flows (less than $10 \mathrm{~m} / \mathrm{s}$ ) but still offers potential for measurements from automotive wind tunnels which can typically be taken at $50 \mathrm{~m} / \mathrm{s}$.

Application of DGV to a large wind tunnel environment and high speed wind tunnels requires a highly refined optical set-up and calibration procedure. This has resulted in initial DGV systems being developed by specialist groups who have matured their systems by incorporating many ongoing improvements [41-44]. These refined systems, however, have allowed DGV measurements to be made in large scale wind tunnels such as the NASA Ames $40 \times 80$ foot [41] and at Mach numbers of up to 0.74 in the ONERA T2 transonic facility [44].

\section{EXPERIMENTAL SET-UP}

The following will now describe the different set-ups for the experimental measurements. These techniques include those for the flow visualisation, the static pressure measurements and the PIV set-up. PIV in this case was the method of choice as it allows rapid acquisition over a plane of interest thus reducing the seeding period which can be an issue in a large wind tunnel volume. Due to it's planar nature, it also records a greater number of data points than LDA over the same time period. Although DGV would relax some of the seeding requirements, however, it is not available at the time of writing as a turn-key solution and thus requires specialist instrumentation knowledge and equipment. 


\subsection{Wind tunnel set-up}

The model notchback car was placed in the closed working section of the Cranfield University $2.4 \mathrm{~m} \times 1.8 \mathrm{~m}$ closed return wind tunnel. The wind tunnel working section has a length of $4 \mathrm{~m}$ and the facility for a rolling road ground simulation with upstream ground plane boundary layer suction. In this case, although the boundary layer suction was activated, the rolling road was not used as the model had fixed wheels and also because all the measurements were completed on the upper surface of the car. The model was secured to the floor at four points via the wheels and aligned with the freestream.

The nominally $1 / 3^{\text {rd }}$ scale model was representative of a modern notchback saloon with a length of $1750 \mathrm{~mm}$. In this case, given the limitations of the wind tunnel, the Reynolds numbers tested and scaled with respect to model length, are listed in table 1.

Thus, within the range of speed allowed by the wind tunnel, the test Reynolds number will be from 3 to 20 times smaller than for the full-scale vehicle. However, given the relatively high Reynolds number in these test cases, it was not expected that the flow structure would be sensitive to changes in Reynolds number within the range allowable.

\subsection{Flow visualisation}

Flow visualisation was carried out in three different ways. Firstly the flow structure behind the rear-window was studied using smoke and illuminated with a laser sheet located along the model centerline. The smoke was introduced at mid-height of the rear window, using a tube coming from the front face of the model and attached to the car surface in such a way that it would cause minimum interference with the flow. The tests were run at relatively low speeds since the smoke entrainment mechanism was only visible for speeds below $18 \mathrm{~m} / \mathrm{s}$. Thus recordings were made for speeds between $U_{\infty}=6$ and $U_{\infty}=18 \mathrm{~m} / \mathrm{s}$, corresponding to Reynolds between $0.74 \times 10^{6}$ $2.22 \times 10^{6}$.

The second flow visualisation method involved attaching short nylon tufts over the rear window and the bootdeck of the model. A camera was then fixed above the test section ceiling overhead the rear of the model in order to record the motions of the tufts when running the tests at different speeds. The tuft distribution is illustrated in Figure 5. The tests were performed for speeds between $U_{\infty}=10 \mathrm{~m} / \mathrm{s}$ and $U_{\infty}=40 \mathrm{~m} / \mathrm{s}$, which correspond to a range of Reynolds numbers between $1.23 \times 10^{6}-4.93 \times 10^{6}$. Between different runs, the wind tunnel was stopped in order to realign the tufts with tunnel axis.

The final method of flow visualisation used surface oil flow in which a fluorescent pigment is suspended in paraffin. During the model preparation, the oil mixture was spread over the model surface at zero wind and the formation of the subsequent surface streamline pattern was recorded using the previous visualisation camera fixed above the test section ceiling. During each test, the wind tunnel was run for sufficient time to allow the oil mixture to dry. Recordings were made at $U_{\infty}=25 \mathrm{~m} / \mathrm{s}$ and $U_{\infty}=40 \mathrm{~m} / \mathrm{s}$, which correspond to Reynolds numbers of $3.09 \times 10^{6}$ and $4.93 \times 10^{6}$ respectively.

\subsection{Static pressure measurements}

A total of 35 surface static pressure tappings were made in the bootdeck area of the model. The tappings were positioned in the backlight, the mid-boot, the end-boot area and along the bootdeck centerline and were located in order to capture steady and unsteady flow features reported by previous workers including the separation bubble and the vortex structures. Figure 6 illustrates these pressure tapping positions on the model where the coordinate origin is positioned at, the model centerline $(x=0)$, the wind tunnel floor $(y=0)$ and the model rear end $(z=0)$.

Each pressure tapping was connected to a port on a Scanivalve head via a $1.1 \mathrm{~m}$ long pneumatic tube with suitable wall stiffness characteristics to facilitate both steady and unsteady pressure measurements. An additional Scanivalve port was also used as an atmospheric reference pressure for all the measurements. 


\subsubsection{Steady pressure measurements}

Preliminary measurements were made at $U_{\infty}=25 \mathrm{~m} / \mathrm{s}, 30 \mathrm{~m} / \mathrm{s}, 35 \mathrm{~m} / \mathrm{s}$ and $40 \mathrm{~m} / \mathrm{s}$, to ensure the suitability of a pressure transducer with a full scale range of $0.1 \mathrm{psi}$. Further tests were completed across a range of transducer sampling frequencies from $400 \mathrm{~Hz}-700 \mathrm{~Hz}$.

The pressure data were corrected to allow for test section blockage of $8 \%$ based on the model maximum cross sectional area. Finally to ensure static pressure measurement insensitivity to boundary layer conditions, two tests were performed: one involving a transition point fixed upstream from the model roof trailing edge and a second one with transition point fixed midlength along the roof. Transition was ensured by attaching a metallic wire (diameter: $0.71 \mathrm{~mm}$ ) across the car width ahead of the roof trailing edge. Measurements under these conditions were performed for speeds within the range $U_{\infty}=25 \mathrm{~m} / \mathrm{s}$ to $U_{\infty}=40 \mathrm{~m} / \mathrm{s}$.

\subsubsection{Unsteady pressure measurements}

In order to correct any potential amplification, attenuation or phase shift of the unsteady pressure signal within the pneumatic tubing system a direct calibration approach was used to estimate the transfer function of the tubes as a function of frequency and static pressure. A single tube was selected and connected to a loudspeaker system driven by a sinusoidal signal both adjustable in frequency and amplitude. Pressure signatures were recorded simultaneously at the entrance of the tube and at the exit of the Scanivalve using a 1 psi differential pressure transducer. The amplitude of the incoming signal was kept constant and the frequency was varied from 10 to 400 $\mathrm{Hz}$ in steps of $10 \mathrm{~Hz}$. The response of the system was then estimated in terms of amplitude ratio and phase.

Figure $7 \mathrm{a}$ shows the equipment used for this process and Figure $7 \mathrm{~b}$ the filter characteristic. When performing unsteady pressure measurements, the Scanivalve and wind tunnel reference transducers were connected to a multi-port amplifier and filter, then to a CED 1401 A-D converter that allowed simultaneous recording of both signals. Thereby, each signal could be independently filtered and/or amplified. Before running the tests, a reference signal was taken at zero wind for both channels. This reference signal was then removed as an offset during data processing. Throughout the test range of velocities of $U_{\infty}=25 \mathrm{~m} / \mathrm{s}-40 \mathrm{~m} / \mathrm{s}$, the wind tunnel was run continuously, changing neither the model configuration nor the Scanivalve pressure transducer. The sample rates and transducer calibration constants are listed in table 2.

\subsection{PIV Set-up}

PIV images were acquired using a TSI Insight 3D stereoscopic PIV system with two TSI Powerview 4MP 12 bit 4 megapixel CCD cameras and a New Wave Solo Nd:YAG double-pulsed laser. Figures 8 and 9 show the basic stereoscopic PIV set-up where the cameras were mounted $1300 \mathrm{~mm}$ behind the rear of the model on a pair of $80 \mathrm{~mm} \times 120 \mathrm{~mm}$ rectangular posts, with an object distance of $1400 \mathrm{~mm}$ corresponding to a camera half angle of $23^{\circ}$. Samples of 200 images were taken for each measurement condition and perspective error [45] eliminated through the use of the stereoscopic set-up. The laser was mounted outside the wind tunnel above the model and projected through a small access hole with the light sheet generated using a set of TSI plano cylindrical and spherical lenses. This resulted in a diverging light sheet of less than $1 \mathrm{~mm}$ thickness illuminating the plane of interest which had dimensions $190 \mathrm{~mm} \times 190 \mathrm{~mm}$ and which was positioned adjacent to the model centerline as shown in Figure 5. The local flow area in the region of interest was seeded using a Fogger smoke generator producing seeding with a size of $0.1 \mu \mathrm{m}-1 \mu \mathrm{m}$. Pulse separations were set to ensure less than $30 \%$ particle image movement through the light sheet. Table 3 below outlines the pulse duration settings.

PIV images were processed from 200 images using the correlation averaging algorithm [46] incorporated into the TSI UltraPIV software. This algorithm has the advantage of yielding velocity data in flows where the seeding quality is highly variable by effectively increasing the signal to noise through the correlation average. A primary correlation window of $64 \times 64$ pixels was selected with a sub-correlation window of $32 \times 32$ pixels with a Gaussian peak search and a validation range of $\pm \mathrm{U}_{\infty}$ and $0.5 \mathrm{U}_{\infty}$ gradient tolerance. Where possible, non-valid vectors were deleted and interpolated using valid data within a $5 \times 5$ averaged matrix. Final results typically yielded 5000 vectors per PIV image corresponding to a spatial resolution of around $2.5 \mathrm{~mm} \times$ 
$2.5 \mathrm{~mm}$ over the imaging area. Expected measurement errors are better than $2 \%$ in plane and $5 \%$ out of plane of full scale velocity. These maximum errors are based on a correlation error of 0,1 pixel and 5 pixel full scale particle image displacement and the imaging geometry corresponding to a camera half angle of around $25^{\circ}[47,48]$. In regions of high seeding density when the number of particle image pairs $N$ is higher, the error in this case would be expected to fall as $1 / \sqrt{ } N$ through the correlation averaging [46]. Further information on correlation errors can be found in a review paper by the first author [49].

\section{RESULTS AND DISCUSSION}

The following section now describes the results from each measurement technique followed by a discussion of findings from the different techniques with comparisons to previous quantitative and qualitative data.

\subsection{Flow visualisation}

The most useful flow visualisation results were from the tuft visualisation and the surface flow visualisation. These results are outlined in more detail below. The smoke flow visualisation was not successful because, at the higher wind tunnel speeds, the smoke was found to dissipate before reaching the bootdeck region. Therefore smoke flow visualization was restricted to the speed range $U_{\infty}=6 \mathrm{~m} / \mathrm{s}-18 \mathrm{~m} / \mathrm{s}\left(\operatorname{Re}=0.74 \times 10^{6}-2.22 \times 10^{6}\right)$ and under these conditions it was difficult to see any coherent structure in the near-wake.

\subsubsection{Tuft flow visualisation}

Tuft flow visualisation allowed approximate observations to be made of the flow structure in the bootdeck area. The three categories of tuft motion seen were: i) tufts remaining aligned with the flow indicating attached flow, ii) tufts which curve and move upstream indicating recirculation areas iii) tufts having rotating motion indicating areas of unsteady flow. On the basis of these criteria, the videos were analysed for all the speeds assessed. It was observed that for speeds below $U_{\infty}=25 \mathrm{~m} / \mathrm{s}$, the near-wake structure appeared weak and the tuft motions did not exhibit any particular features. However, analysis of the videos from $U_{\infty}=25 \mathrm{~m} / \mathrm{s}$ to $U_{\infty}=40 \mathrm{~m} / \mathrm{s}$ led to an assessment of the flow field as listed in Table 4 and illustrated in Figure 10.

Given these observations, some general comments can be made with respect to the bootdeck flow structures. Firstly, that the shear layer appears to reattach onto the bootdeck, as has been observed by previous workers [6]. Secondly, the unsteadiness appears to move from left to right depending on the speed, which indicates that the flow structure may be asymmetric and varying with Reynolds number.

\subsubsection{Surface flow visualisation}

Surface flow visualization was carried out at $U_{\infty}=25 \mathrm{~m} / \mathrm{s}\left(R e=3.09 \times 10^{6}\right)$ and $U_{\infty}=40 \mathrm{~m} / \mathrm{s}(R e=$ $\left.4.93 \times 10^{6}\right)$. To provide adequate drying of the fluorescent flow visualization mixture on the surface, the flow visualisation pictures were taken approximately 9 minutes after the beginning of each test.

Results from the $U_{\infty}=25 \mathrm{~m} / \mathrm{s}$ test are shown in Figure 11a. There are four major flow features of interest at this flow speed. Points 1 indicate the presence of two reattachment nodes at the downstream edge of the hairpin vortex region. The left and right hand sides of these two reattachment regions do not appear to have the same corresponding positions along the same point of the bootdeck therefore indicating a degree of flow asymmetry. Points 2 represent two unstable focus areas, with their instability partly recognisable by the poor distribution of the visualization material on the surface. These two focus areas are expected to yield two major contra-rotating vortical structures which extend over the bootdeck region as indicated by the surface flow lines extending out from point 1 on each side. Point 3 is unclear due to the poor residual flow visualisation material. However, given the presence of the two adjacent hairpin vortices, this point is expected to be a form of saddle point with the flow extending out from the 
backlight and then over the bootdeck region. Point 4 although not definitive, appears to be near a separation line, probably generated by the interaction of two contra-rotating streamwise vortices which originate from the hairpin vortices of points 2 . Although Figure $8 \mathrm{a}$ shows the flow pattern to be asymmetric, the general flow structure seems to correlate well with that proposed by Gilhome [6] from his own surface flow visualization (see Figure 1 for comparison). The separation lines and saddle point, however, which are just detectable on the left hand side of the model, and undetectable on the right hand side, may modify part of the surface flow structure pattern. However, in general the major structures expected, appear to be visible.

Figure $11 \mathrm{~b}$ shows results from the $\mathrm{U}_{\infty}=40 \mathrm{~m} / \mathrm{s}$ test. As with the previous example, the major points of interest are shown on the image. These results when compared to the $U_{\infty}=25 \mathrm{~m} / \mathrm{s}$ test appear to have similar flow structures except that the flow structure is a mirror image about the middle centerline of the lower speed test case with the corresponding positions of the points of interest located on the opposite side of the model. This change in geometry was also noted in the tuft flow visualisation results. One other minor difference between the two test cases is also the possible presence of a small focus point on the right hand reattachment node point 1 . Similar degrees of unsteadiness appear to be present as found in the $U_{\infty}=25 \mathrm{~m} / \mathrm{s}$ case based on the distribution of the flow visualization material on the surface. With these limited results, however, it is difficult to offer a conclusive explanation for why the flow structure appears to move and why vortices change their relative location given that the flow would be expected to be independent of Reynolds number. The flow structure in the near wake may be bi-stable, the asymmetry being driven by very small changes in model orientation, or 'velocity history' in terms of the run-up to speed, rather than by a conventional Reynolds number related mechanism.

\subsection{Particle Image Velocimetry}

PIV data was recorded from the model over the speed range $U_{\infty}=20 \mathrm{~m} / \mathrm{s}-30 \mathrm{~m} / \mathrm{s}$ with 200 image pairs per run. Time-averaged vector maps could only be generated from the PIV images as sufficient seeding levels could not be obtained for satisfactory full-field instantaneous PIV data. The low seeding levels therefore led to the use of the correlation averaging routine to yield full field time-averaged data for all the flow cases studied.

Figures 12 and 13 show the 3D stereoscopic PIV vector maps. In this case the out in-plane components $u$ and $v$ are represented by the vector map with additional large scale arrows to clarify general flow structure. The out of plane component $w$ is represented by the colour contour plots superimposed onto the vector plots. In this case red represents velocity in the freestream direction, green stationary flow and blue reversed flow.

Figure 12 shows $3 \mathrm{D}$ vector plots for three lateral planes between the backlight and the rear boot (see Figure 6) at a free stream velocity of $30 \mathrm{~m} / \mathrm{s}$. All three plots show the dominance of the freestream component on the data with out-of-plane velocities of the order of $30 \mathrm{~m} / \mathrm{s}$ covering the majority of the measurement plane. At the mid-boot position (Figure 12b), flow structure leading to possible flow separation is visible to the left hand side of the flow field through lower $w$ component velocities and the modified in-plane flow structure. However the flow has not reversed at this point which would be definitive of full flow separation. The remaining lower velocity regions adjacent to backlight surface are attributed to reflections off the model surface causing a poor signal to noise ratio and resultant large errors. These reflective effects were reduced by painting the model surface matt black, but were never completely eliminated. In the rear-boot plane, the freestream component is still dominant but over a smaller area as it appears that separation effects, in terms of the expected hairpin and rear-pillar vortices, become established over the boot region. Therefore with reference to Figure 1, it is likely that the PIV measurement planes have been positioned adjacent either the hairpin or rear-pillar recirculation structures. This result thus infers that if present the hairpin vortex would be positioned less than $x / L<0.04$ and the rear-pillar vortex at $x / L>0.15$ at the rear-boot region. Further stereoscopic measurements either side of the current region of interest would be required to confirm this finding.

Figure 13 shows 3D PIV time-averaged data from one plane of interest at the end-boot position taken at three different velocities to investigate the effect of changes in Reynolds number on the flow structures over the car. The results were taken at wind speeds between $20 \mathrm{~m} / \mathrm{s}-30 \mathrm{~m} / \mathrm{s}$ corresponding to Reynolds numbers ranging from $2.47 \times 10^{6}-3.69 \times 10^{6}$. Initial inspection of the results shows the dominance of the freestream component in all three cases through the majority 
of the area of interest. There also appears to be little effect of Reynolds number on the overall flow structure. In all three cases, there is also a greater horizontal component of flow structure for $\mathrm{x} / \mathrm{L}>0.1$ which may be attributed to the effect of the rear-pillar vortex adjacent to the PIV regions of interest. In all three cases, however, the dominant velocity component is clearly the freestream (w) direction which also indicates this region of interest is less affected by any adjacent separation structures. It should be noted that this Reynolds number insensitivity contradicts a number of the findings of the flow visualization studies, but actually reinforces findings of the static pressure studies outlined in the following section. Also these tests were undertaken during a different test phase to that of the flow visualization and as a result minor changes in model orientation cannot be ruled out. If the flow were to be particularly sensitive to such changes this may explain the differences.

\subsection{Static pressure measurements}

The static pressure measurements can be broken down into two parts. These are the steady and unsteady pressure measurements. These results will now be outlined in more detail.

\subsubsection{Steady static pressure measurements}

Figures $14 \mathrm{a}, 14 \mathrm{~b}$ and $14 \mathrm{c}$ show the steady static pressure coefficient $\left(\mathrm{c}_{\mathrm{p}}\right)$ measurements at the backlight, mid-boot and end-boot $z$ measurement planes for freestream velocities between $U_{\infty}=$ $25 \mathrm{~m} / \mathrm{s}-40 \mathrm{~m} / \mathrm{s}$. The results show the flow to be initially almost symmetrical in the $x$ profile in the backlight region, but the profiles becoming more distorted towards the rear of the car in the midboot and end-boot regions. The $c_{p}$ data also becomes more positive in the central region towards the rear of the bootdeck which indicates velocities lower than freestream in the hairpin vortex and freestream vortex regions. Also towards the side of the bootdeck, the $c_{p}$ level drops indicating acceleration of the flow in the rear-pillar vortex region in the end-boot region decreasing from $c_{p}=$ -0.17 to $c_{p}=-0.22$.

The distorted $c_{p}$ characteristic in the rear-boot unfortunately contradicts the general findings of the surface flow visualisation which found an asymmetrical pattern on the surface in this backlight region. However, there was also an additional contradiction between these results as the steady $c_{p}$ pressure data shows minimal sensitivity to Reynolds number over the range of flow speeds with little change in the distorted $c_{p}$ distribution. This is in contrast to the flow visualisation data which indicates changes in the backlight flow structure between the two test cases of $U_{\infty}=25 \mathrm{~m} / \mathrm{s}$ and $\mathrm{U}_{\infty}=40 \mathrm{~m} / \mathrm{s}$.

\subsubsection{Unsteady static pressure measurements}

Figure 15 shows the rms $c_{p}$ unsteady pressure distributions at the backlight, mid-boot and endboot $z$ measurement planes for freestream velocities between $U_{\infty}=25 \mathrm{~m} / \mathrm{s}-40 \mathrm{~m} / \mathrm{s}$. The general trend in all three measurement planes show increased unsteadiness towards the edges of the bootdeck. This would be expected as these regions correspond to the rear-pillar vortex flow structures which will be highly turbulent. There is also a significant fall in the rms $c_{p}$ towards the centre of the bootdeck particularly at the mid-bootdeck region. This indicates that the flow becomes more stable as it develops over the bootdeck and leaves the rear of the model.

Consideration of the effect of Reynolds number results in the following observations. Generally over the range of Reynolds number tested, between $R e=3.08 \times 10^{6}-4.93 \times 10^{6}$, there was no obvious change in the overall level of $c_{p}$ fluctuations, particularly in the backlight region. This result was reinforced by the spectral results of selected pressure tappings where there appeared to be no dominant modes at any of the planes through the range of Reynolds numbers tested. A typical example of these broadband characteristics can be seen in Figure 16 which shows spectral data at the end-boot centreline position at $40 \mathrm{~m} / \mathrm{s}$. Further examination of the spectra at different positions and conditions also gave similar findings for Reynolds number insensitivity. This result confirms findings from the PIV data and rms pressure data. 


\section{CONCLUSIONS}

Flow visualisation, PIV and static pressure results have been presented from wind tunnel studies of the bootdeck region of a $36 \%$ scale model of a notchback saloon car. The flow visualisation results have indicated flow asymmetries in the backlight region which appear to be sensitive to changes in freestream speed. These asymmetries are likely to originate from two unstable focus points either side of the backlight with a bi-stable near wake flow structure which is particularly sensitive to external factors within this Reynolds number range. In contrast, further investigation of the flow in the rear bootdeck area during a subsequent test programme with stereoscopic PIV suggested little or no sensitivity of the flow structure to Reynolds number. The 3D PIV timeaveraged data also seems to have been positioned between hairpin and rear-pillar vortex structures as no significant flow features were found from this data. Thus the rear-pillar and hairpin vortex structures are expected to be positioned at $x / L<0.04$ and $x / L>0.15$ respectively. Steady and unsteady static pressure data also showed a Reynolds number insensitive flow structure from the backlight to the rear bootdeck area with a nearly symmetric $c_{p}$ profile at the rear of the car. In addition unsteady measurements showed no dominant frequency to exist through the range of Reynolds numbers and bootdeck positions tested. Discrepancies between the flow visualisation and the other flow data can be in part attributed to difficulty in interpreting the flow visualisation in the backlight region. Further work is now planned using 3D stereoscopic time-resolved PIV (TR-PIV) over the centreline and boot edge regions to confirm the presence of the hairpin and rear-pillar structures and ascertain any unsteady characteristics or Reynolds number sensitivity in these regions. Such a study will require significant improvements to the seeding levels introduced into the flow region of interest. 


\section{REFERENCES}

1. Katz, J. Race Car Aerodynamics - Designing for Speed. Bentley Publishers, 1995, ISBN 08376-0142-8.

2. Carr, G.W. (1975). Correlation of pressure measurements in model and full-scale wind tunnels and on the road. Society of Automotive Engineers, SAE 750065

3. Nouzawa, T., Hiasa, K. and Nakamura, T. (1990). Analysis of wake pattern for reducing aerodynamic drag of notchback model. Society of Automotive Engineers, SAE 900318

4. Jenkins, L.N. (2000). An experimental investigation of the flow over the rear end of a notchback automobile configuration. Society of Automotive Engineers, SAE 2000-01-0489

5. Carr, G.W. (1974). Influence of rear body shape on the aerodynamic characteristics of saloon cars. MIRA 1974/2

6. Gilhome, B.R., Saunders, J.W and Sheridan, J. (2001). Time averaged and unsteady nearwake analysis of cars. Society of Automotive Engineers, SAE 2001-01-1040

7. Gilhome, B.R. (2002). Unsteady flow structures and forces over/on the rear window and boot lid of sedan automobiles. In press

8. Adrian, R.J. (1991), Particle-Imaging techniques for experimental fluid mechanics, Annual Review of Fluid Mechanics, Vol. 23, pp. 261-304

9. Pickering, C.J.D and Halliwell, N.A. Speckle photography in fluid flows: signal recovery with two step processing. Applied Optics, 1984, 23(8), 1128

10. Drain, L.E. The laser Doppler technique. John Wiley \& Sons (Chichester), 1980.

11. Durst, F., Melling, A. and Whitelaw, J.H. Principles and practice of laser Doppler anemometry. Academic Press (London), 1981.

12. Albrecht, H.-E., Damaschke, N., Borys, M. and Tropea, C. Laser Doppler and phase Doppler measurement techniques. Springer (Berlin), 2002.

13. Wigley, G. Phase Doppler anemometry and its application to liquid fuel spray combustion. Optical Diagnostics for Flow Processes (Eds L. Lading, G. Wigley and P. Buchave), 1994, pp175-204 (Plenum, New York)

14. Jeffrey, D., Zhang, X. and Hurst, D.W. Aerodynamics of Gurney flaps on a single-element high-lift wing. Journal of Aircraft, 2000, 37(2), 295-301.

15. Raffel, M., Seelhorst, U. and Willert, C. Vortical flow structures at a helicopter rotor model measured by LDV and PIV. The Aeronautical Journal, 1998, Paper 2227, 221-227.

16. Dudderar, T.D. and Simpkins, P.G. Laser speckle photography in a fluid medium. Nature, 1977, 270, 45-47.

17. Prasad, A.K. Stereoscopic particle image velocimetry. Experiments in Fluids, 2000, 29, 103116.

18. Brucker, Ch. 3D Scanning PIV applied to an air flow in a motored engine using digital high speed video. Measurement Science and Technology, 1997, 8, 1480-1492. 
19. Willert, C.E. and Gharib, M. Three-dimensional particle imaging with a single camera. Experiments in Fluids, 1992, 12, 353-358.

20. Coupland, J.M., Halliwell, N.A. Particle image velocimetry: three-dimensional fluid velocity measurements using holographic recording and optical correlation. Applied Optics, 1992, 31, 1005-1007.

21. Barnhart, D.H., Adrian, R.J. and Papen, G.C. Phase-conjugate holographic system for high resolution particle image velocimetry. Applied Optics, 1994, 33(30), 7159-7170.

22. Kawanabe, H., Kawasaki, K. and Masahiro, S. Gas-flow measurements in a jet flame using cross-correlation of high-speed-particle images. Measurement Science Technology, 2000, $11,627-632$.

23. Reeves, M., Towers, D.P., Tavender, B. and Buckberry, C.H. A high-speed all-digital technique for cycle-resolved 2-D flow measurement and flow visualisation within SI engine cylinders. Optics and Lasers in Engineering, 1999, 31(4), 247-261.

24. Raffel, R., Willert, C.E. and Kompenhans, J. Particle image velocimetry: a practical guide. Springer-Verlag, Berlin, 1997.

25. Willert, C., Raffel, M., Kompenhans, J., Stasicki, B. and Kahler, K. Recent applications of particle image velocimetry in aerodynamic research. Flow Measurement Instrumentation, 1996, 7(3/4), 247-256.

26. Kooi, J.W., Pengel, K., Raffel, M., Willert, C., Kompenhans, J. Application of PIV in the large low speed facility of DNW. AGARD FDP Symposium on "Advanced Aerodynamic Measurement Technology", Seattle 22-25 September 1997, 1997, paper CP-601.

27. Yeung, A.F.K. and Lee, B.H.K. Particle image velocimetry study of wing tip vortices. Journal of Aircraft, 1999, 36(2), p482-484.

28. Early, J.M., Green, R.B. and Coton, F.N. Flow visualisation of the orthogonal blade-vortex interaction using particle image velocimetry. The Aeronautical Journal, 2002, Paper 2635, 137-145.

29. McCutcheon, G., McColgan, A.H., Grant, I. and Hurst, H. Wake studies of a model passenger car using PIV. Proceedings of the 2002 SAE Motorsports Engineering Conference and Exhibition, 2002, Paper 2002-01-3335, 487-496.

30. Miles, R.B and Lempert, W.R. Flow diagnostics in unseeded air. AlAA Paper 90-0624, 1990

31. Komine, H., Brosnan, S.J., Litton, A.B., Stappaerts, E.A. Real-time, Doppler global velocimetry. AIAA Paper 91-0337, 1991.

32. Meyers, J.F. Development of Doppler global velocimetry as a flow diagnostics tool. Measurement Science Technology, 1995, 6, 769-783.

33. Beutner T.J., Baust, H.D. Recent developments in Doppler global velocimetry. AGARD Conference Proceedings 601 - Advanced Aerodynamic Measurement Technology, 1998, AGARD-CP-601, 8.1-8.9.

34. Beutner, T.J., Elliot, E., Crafton, J. and Carter, C.D. Characterization and applications of Doppler global Doppler velocimetry. $37^{\text {th }}$ Aerospace Sciences Meeting and Exhibit, 11-14 January 1999, Reno, 1999, AIAA Paper 99-0266, 1-21. 
35. Elliot S.E. and Beutner T.J. Molecular filter based planar Doppler velocimetry. Progress in Aerospace Sciences, 1999, 35, 799-845.

36. Elliot, G.S., Beutner, T.J. and Carter, C.D. Application of planar Doppler velocimetry wind tunnel testing. $38^{\text {th }}$ Aerospace Sciences Meeting and Exhibit, 10-13 January 2000, Reno, 2000, AIAA Paper 2000-0412, 1-35.

37. Willert, C., Roehle, I., Schodl, R., Dingel, O. and Seidel, T. Application of planar Doppler velocimetry within piston engine cylinders. $11^{\text {th }}$ International Symposium on Applications of Laser Techniques to Fluid Mechanics, Lisbon, July 8-1 $1^{\text {th }}$ 2002, 2002, Paper 38.2, 1-10.

38. Chan, V.S.S., Heyes, A.L., Robinson, D.I. and Turner, J.T. lodine absorption filters for Doppler global velocimetry. Measurement Science Technology, 1995, 6, 784-794.

39. Meyers, J.F. and Joseph, W.L. Identification and minimisation of errors in Doppler global velocimetry measurements. $10^{\text {th }}$ International Symposium on Applications of Laser Techniques to Fluid Mechanics, Lisbon, July 10-13 ${ }^{\text {th }} 2000,2000$, Paper 35.1, 1-12.

40. Meyers, J.F, Joseph, W.L. and Schwartz, R.J. Characterization of measurement error sources in Doppler global velocimetry. Measurement Science Technology, 2001, 12, 357368.

41. Meyers, J.F, Lee, J.W., Fletcher, M.T and Bruce, W.S. Hardening Doppler global velocimetry systems for large wind tunnel applications. AIAA Paper 98-2606, 1998, 1-14.

42. Beutner, T.J., Elliot, E., Mosedale, A., and Carter, C.D. Doppler global velocimetry applications in large scale facilities. $20^{\text {th }}$ AIAA Advanced Measurement Technology and Ground Testing Conference, 15-18 June 1998, Albuqerque, 1998, AIAA Paper 98-2608, 120.

43. Beutner, T.J., Elliot, E., Williams, G.W., Baust, H.D., Crafton, J. and Carter, C.D. Forebody and leading edge vortex measurements using planar Doppler velocimetry. Measurement Science Technology, 12, 2001, 378-394.

44. Barricau, P., Lempereur, C., Mathe, J.M, Mignosi, A. and Buchet, H. Doppler global velocimetry: development and wind tunnel tests. $11^{\text {th }}$ International Symposium on Applications of Laser Techniques to Fluid Mechanics, Lisbon, July 8-1 ${ }^{\text {th }}$ 2002, 2002, Paper 9.4, 1-12.

45. Reeves, M. and Lawson, N.J. (2004), Evaluation and correction of perspective errors in endoscopic PIV, Experiments in Fluids 36 (2004) 701-705

46. Meinhart, C.D, Wereley, S.T. and Santiago, J.G. A PIV algorithm for estimating timeaveraged velocity fields. Journal of Fluids Engineering, 2000, 122, 285-289.

47. Westerweel, J. Fundamentals of digital particle image velocimetry. Measurement Science Technology, 1997, 8, 1379-1392.

48. Lawson, N.J., Wu, J. Three-Dimensional Particle Image Velocimetry: Error Analysis of Stereoscopic Techniques. Measurement Science and Technology, 1997, 8, 894-900.

49. Lawson, N.J., The application of laser measurement techniques to aerospace flows. Journal of Aerospace Engineering 2004, 218, Part G4, p33-57 


\begin{tabular}{|c|c|}
\hline Test speed & Reynolds number \\
\hline $6 \mathrm{~m} / \mathrm{s}$ & $0.74 \times 10^{6}$ \\
\hline $20 \mathrm{~m} / \mathrm{s}$ & $2.47 \times 10^{6}$ \\
\hline $25 \mathrm{~m} / \mathrm{s}$ & $3.08 \times 10^{6}$ \\
\hline $30 \mathrm{~m} / \mathrm{s}$ & $3.69 \times 10^{6}$ \\
\hline $35 \mathrm{~m} / \mathrm{s}$ & $4.31 \times 10^{6}$ \\
\hline $40 \mathrm{~m} / \mathrm{s}$ & $4.93 \times 10^{6}$ \\
\hline
\end{tabular}

Table 1: Wind tunnel test conditions and Reynolds numbers

\begin{tabular}{|l|l|l|l|}
\hline Wind Tunnel reference pressure transducer & \multicolumn{2}{|c|}{ Scanivalve pressure transducer } \\
\hline Calibration & $01.05 \mathrm{mmH}_{2} \mathrm{O} / \mathrm{V}$ & Calibration & $29.895 \mathrm{mmH}_{2} \mathrm{O} / \mathrm{V}$ \\
\hline Amplifier gain & 1 & Amplifier gain & 1 \\
\hline Filter & $0.1 \mathrm{kHz}$ & Filter & $10 \mathrm{kHz}$ \\
\hline Sampling frequency & $3 \mathrm{kHz}$ & Sampling frequency & $3 \mathrm{kHz}$ \\
\hline Sampling duration & 10 seconds & Sampling duration & 10 seconds \\
\hline
\end{tabular}

Table 2: Static pressure measurement configuration and calibration

\begin{tabular}{|c|c|}
\hline Test speed $(\mathrm{m} / \mathrm{s})$ & $\begin{array}{c}\text { PIV laser pulse separation } \\
(\mu \mathrm{s})\end{array}$ \\
\hline 20 & 37.5 \\
\hline 25 & 30 \\
\hline 30 & 25 \\
\hline
\end{tabular}

Table 3: PIV pulse separation settings

\begin{tabular}{|c|c|c|c|}
\hline $\begin{array}{c}\text { Test } \\
\text { speed }\end{array}$ & Re & $\begin{array}{c}\text { Shear layer } \\
\text { reattachment }\end{array}$ & $\begin{array}{c}\text { Bootdeck unsteadiness } \\
\text { localisation }\end{array}$ \\
\hline $25 \mathrm{~m} / \mathrm{s}$ & $3.08 \times 10^{6}$ & Yes & Left hand side \\
\hline $30 \mathrm{~m} / \mathrm{s}$ & $3.69 \times 10^{6}$ & Yes & Right hand side \\
\hline $35 \mathrm{~m} / \mathrm{s}$ & $4.31 \times 10^{6}$ & Yes & Right hand side \\
\hline $40 \mathrm{~m} / \mathrm{s}$ & $4.93 \times 10^{6}$ & Yes & Left hand side \\
\hline
\end{tabular}

Table 4: Tuft visualisation results summary 


\section{Figures}

Figure 1: Time-averaged flow structure proposed by Gilhome [6]

Figure 2 - Schematic of 3D laser Doppler anemometry system

Figure 3 - Schematic of PIV measurement system

Figure 4 - Measurement components in a DGV system

Figure 5 - Overhead view of typical nylon tuft distribution over the model rear surface, flow from left to right

Figure 6 - Relative locations of surface static pressure tappings and PIV measurement planes over the model rear body.

Figure $7-$ a) Experimental arrangement for tubing system calibration b) Filter characteristics

Figure 8 - Experimental arrangement for stereoscopic PIV measurements, top sketch rearview looking upwind, bottom sketch planview (flow from top to bottom)

Figure 9 - View of stereoscopic PIV system with calibration grid

Figure 10 - Surface nylon tuft flow visualisation observations over model rearbody at different freestream speeds (flow from top to bottom in photographs)

Figure 11 - Surface flow visualisation observations on model rearbody a) $25 \mathrm{~m} / \mathrm{s}\left(\operatorname{Re}=3.08 \times 10^{6}\right)$ b) $40 \mathrm{~m} / \mathrm{s}\left(\operatorname{Re}=4.93 \times 10^{6}\right)$ - freestream flow direction from top to bottom

Figure $12-3 \mathrm{D}$ PIV data at $\mathrm{V}_{\infty}=30 \mathrm{~m} / \mathrm{s}\left(\mathrm{Re}=3.69 \times 10^{6}\right)$ a) backlight b) mid-boot $\mathrm{c}$ ) rear boot

Figure 13 - 3D PIV Data at end-boot position a) $V_{\infty}=20 \mathrm{~m} / \mathrm{s}\left(R e=2.47 \times 10^{6}\right)$ b) $V_{\infty}=25 \mathrm{~m} / \mathrm{s}(R e=$ $\left.3.08 \times 10^{6}\right)$ b) $V_{\infty}=30 \mathrm{~m} / \mathrm{s}\left(\operatorname{Re}=3.69 \times 10^{6}\right)$

Figure $14-$ a) $c_{p}$ steady pressure distributions for the bootdeck region a) backlight region b) midboot region c) end-boot region

Figure $15-a) c_{p}$ unsteady pressure distributions for the bootdeck region a) backlight region b) mid-boot region $c$ ) end-boot region

Figure 16 - spectral distribution from the end-boot region $\left(V_{\infty}=40 \mathrm{~m} / \mathrm{s}, \mathrm{Re}=4.93 \times 10^{6}, \mathrm{x} / \mathrm{L}=0\right)$ 


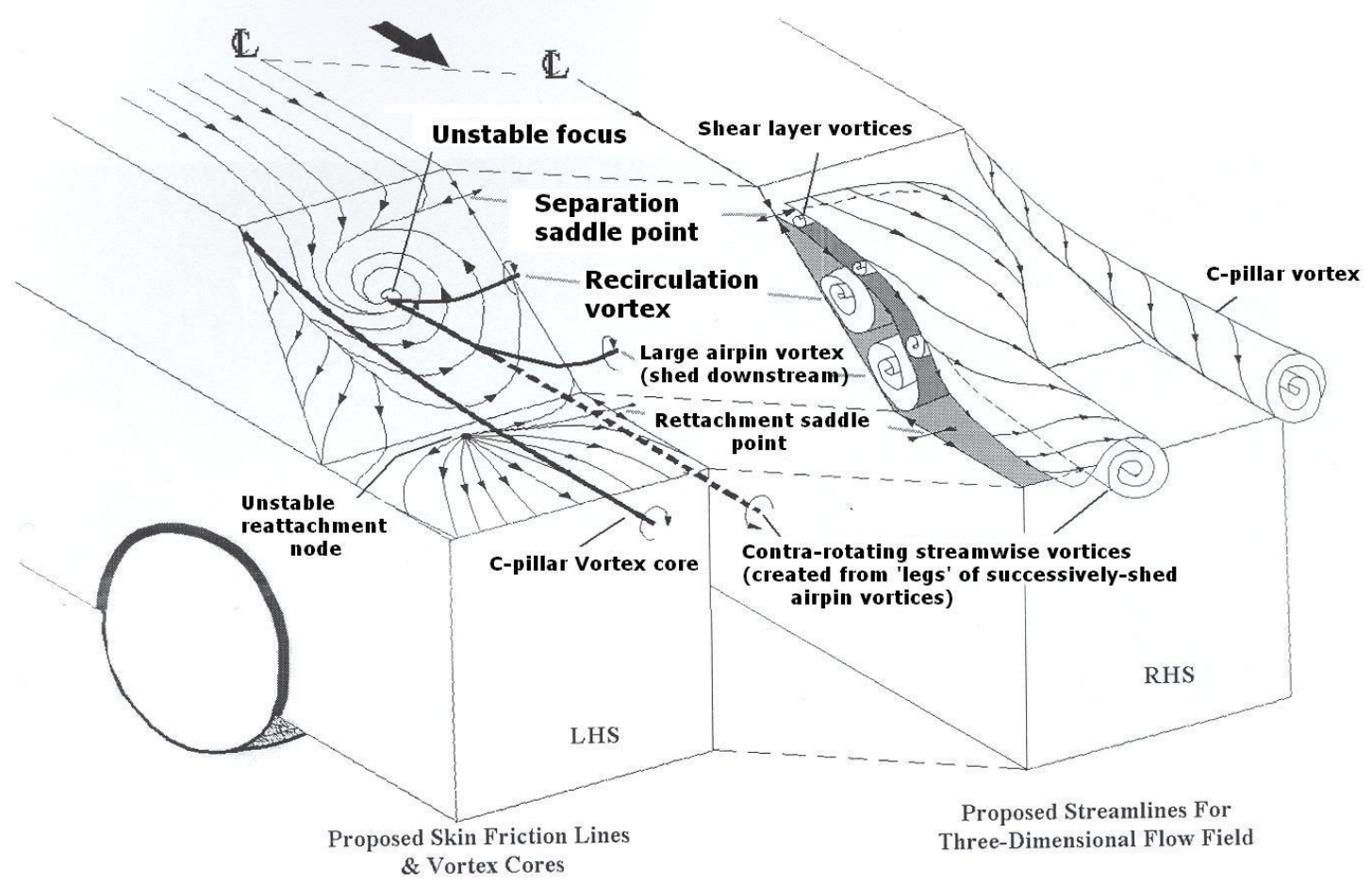

Figure 1: Time-averaged flow structure proposed by Gilhome [6] 


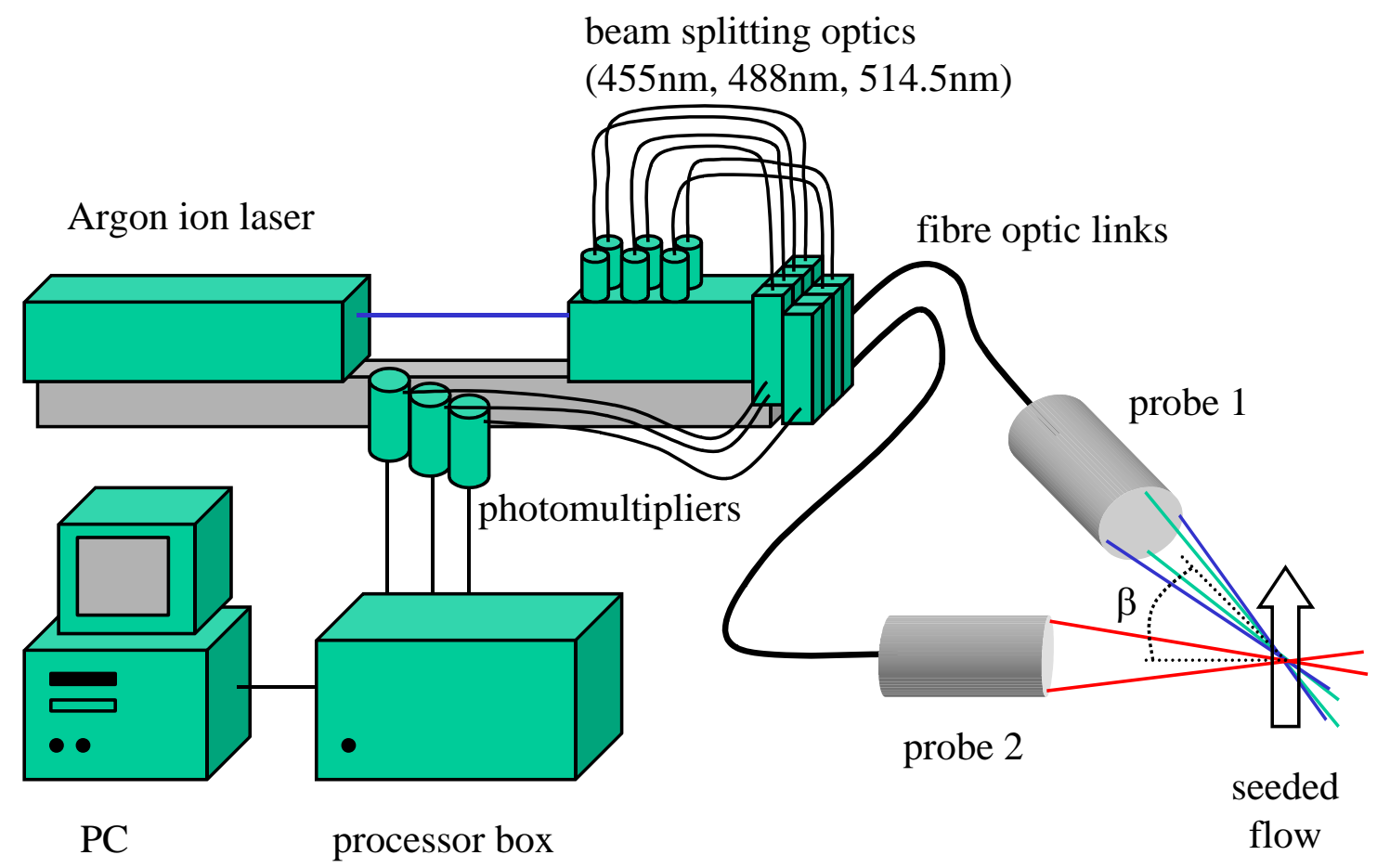

Figure 2 - Schematic of 3D laser Doppler anemometry system 


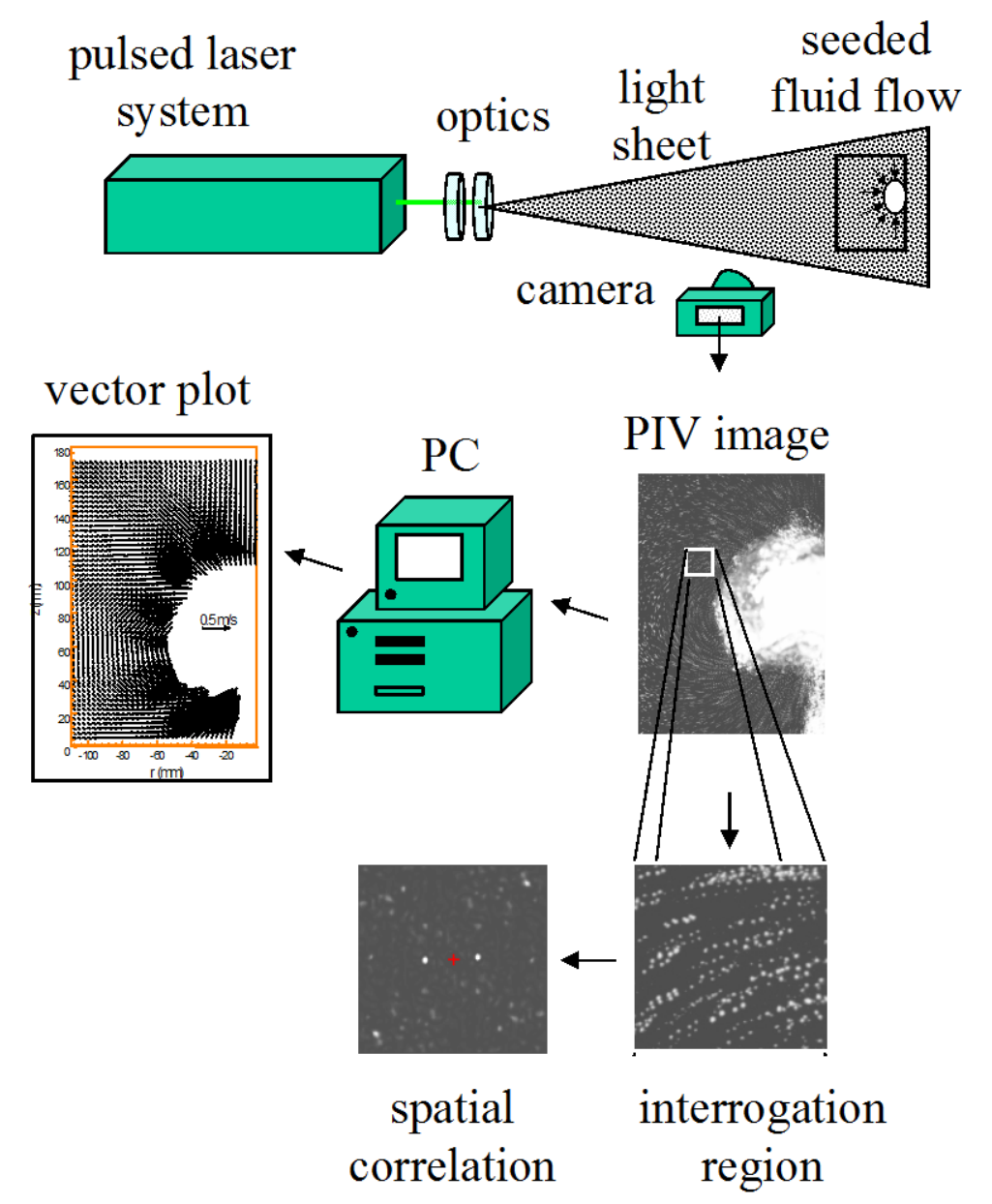

Figure 3 - Schematic of PIV measurement system 


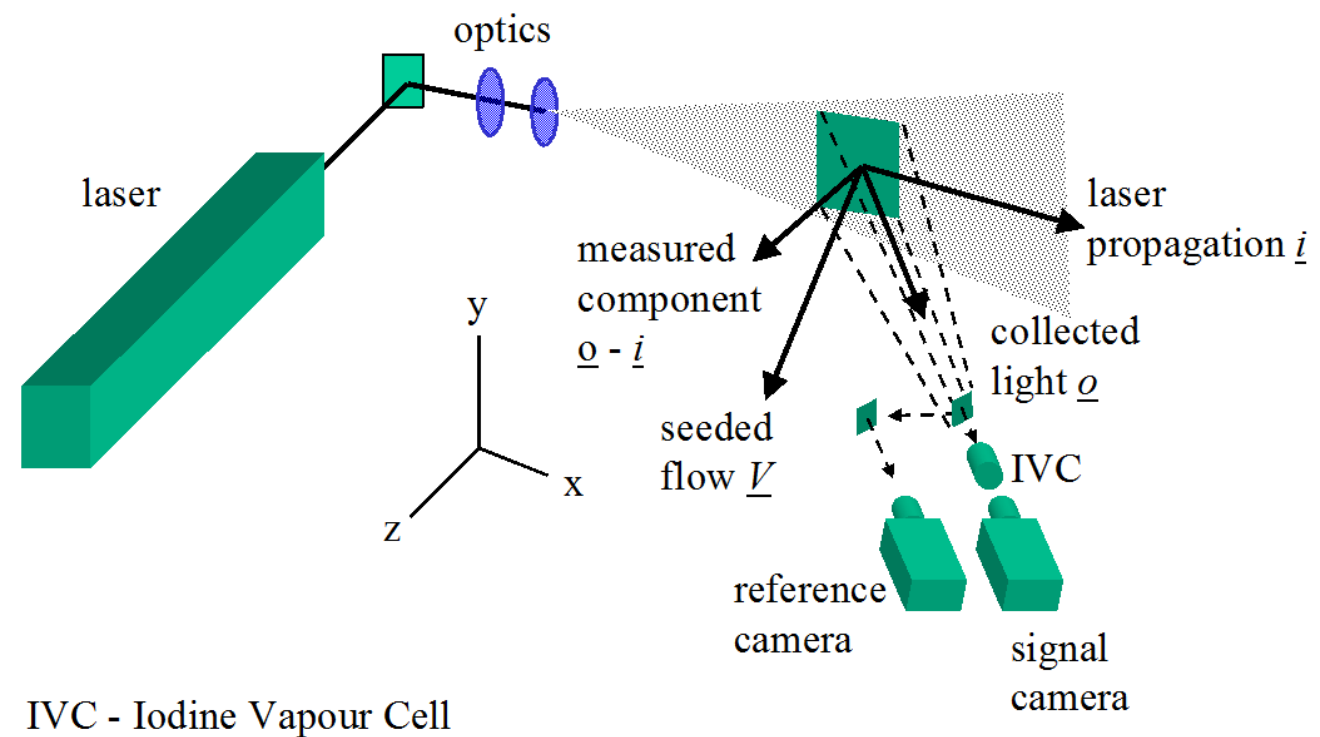

Figure 4 - Measurement components in a DGV system 


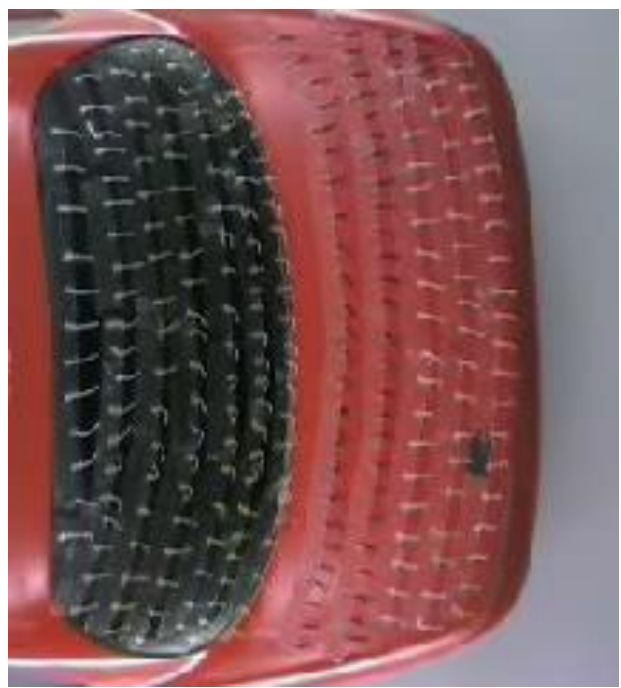

Figure 5 - Overhead view of typical nylon tuft distribution over the model rear surface, flow from left to right

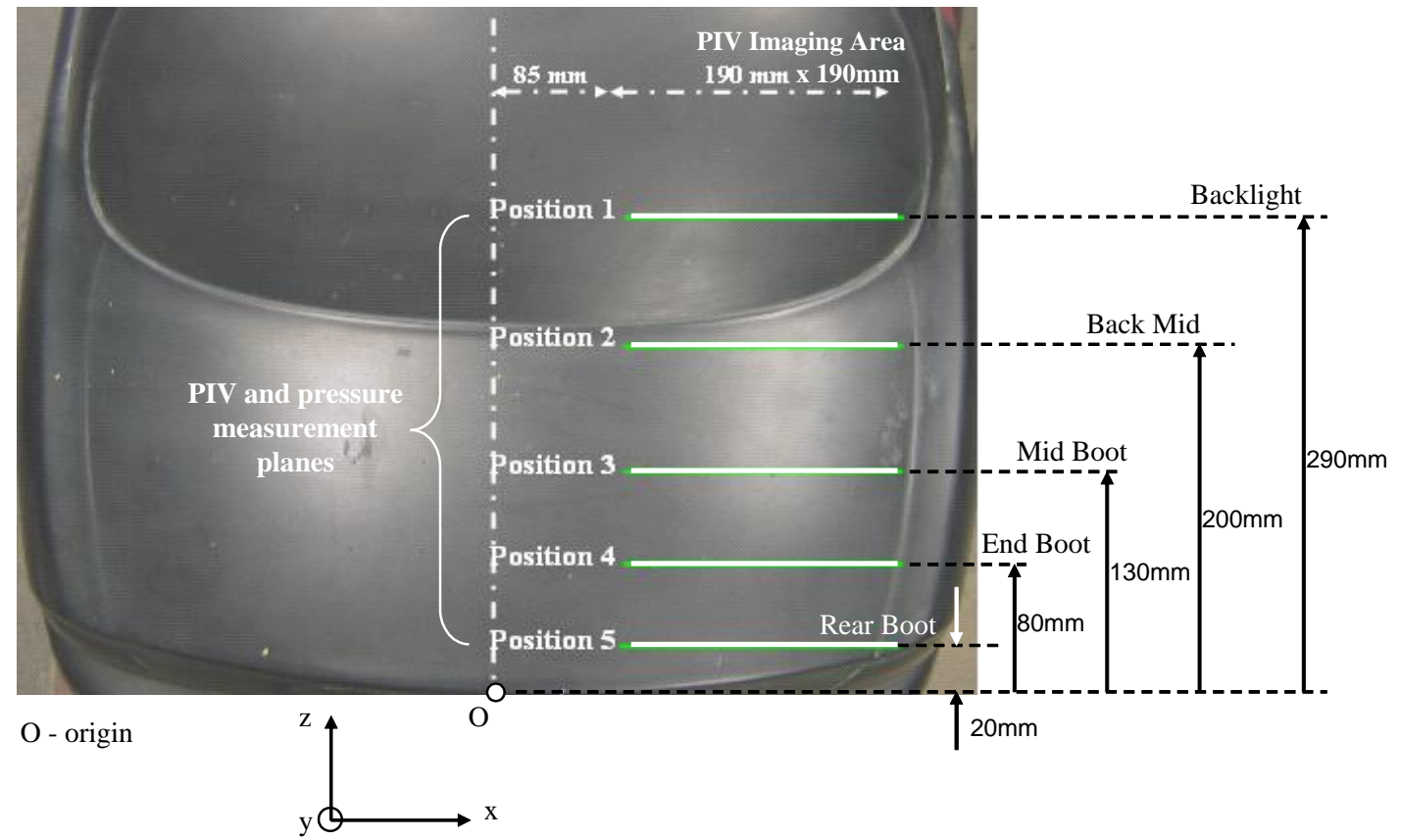

Figure 6 - Relative locations of surface static pressure tappings and PIV measurement planes over the model rear body. 


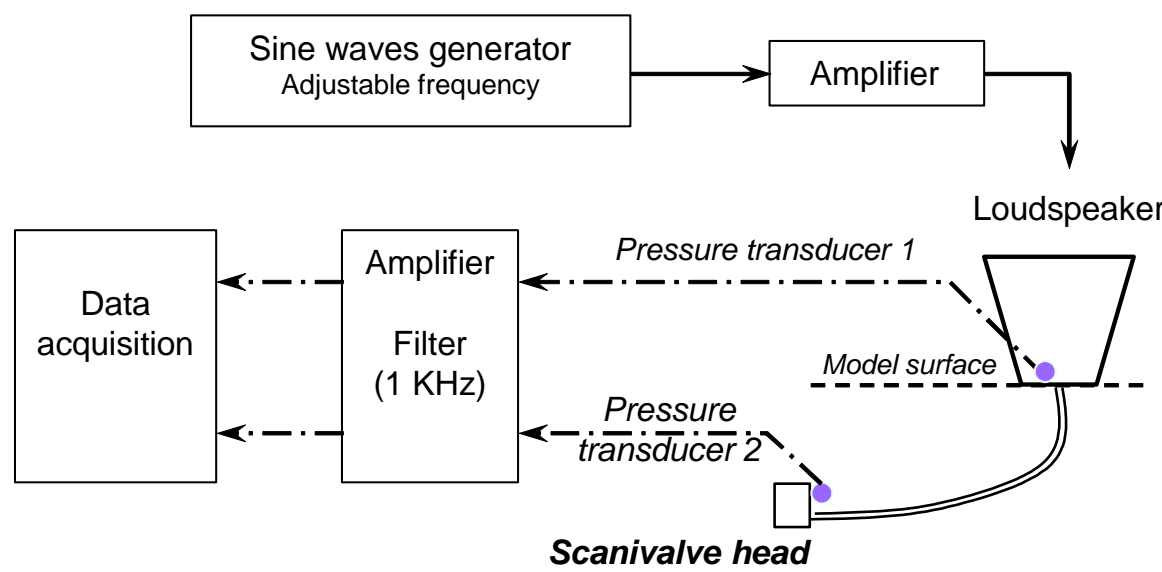

a)

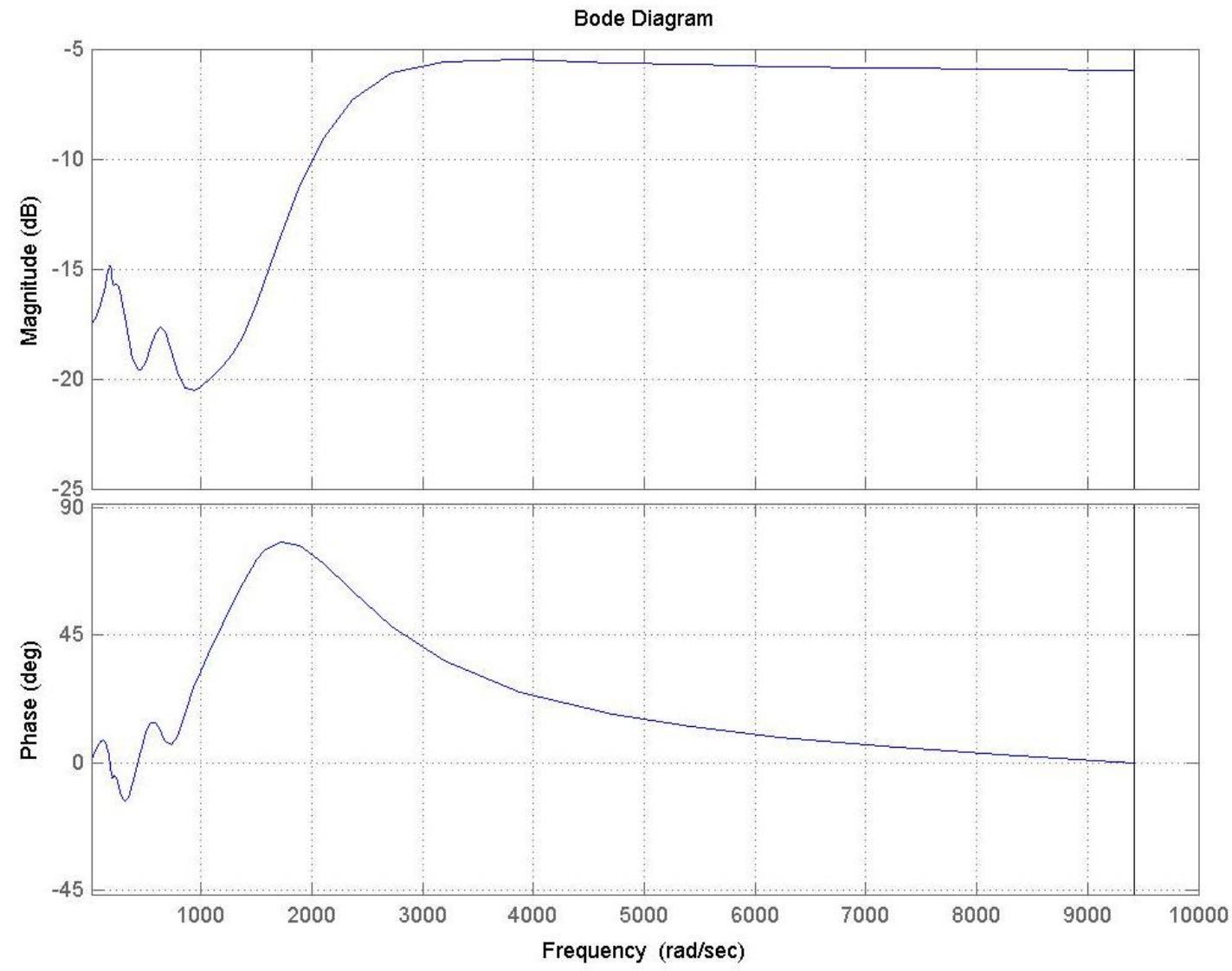

b)

Figure 7 - a) Experimental arrangement for tubing system calibration b) Filter characteristics 


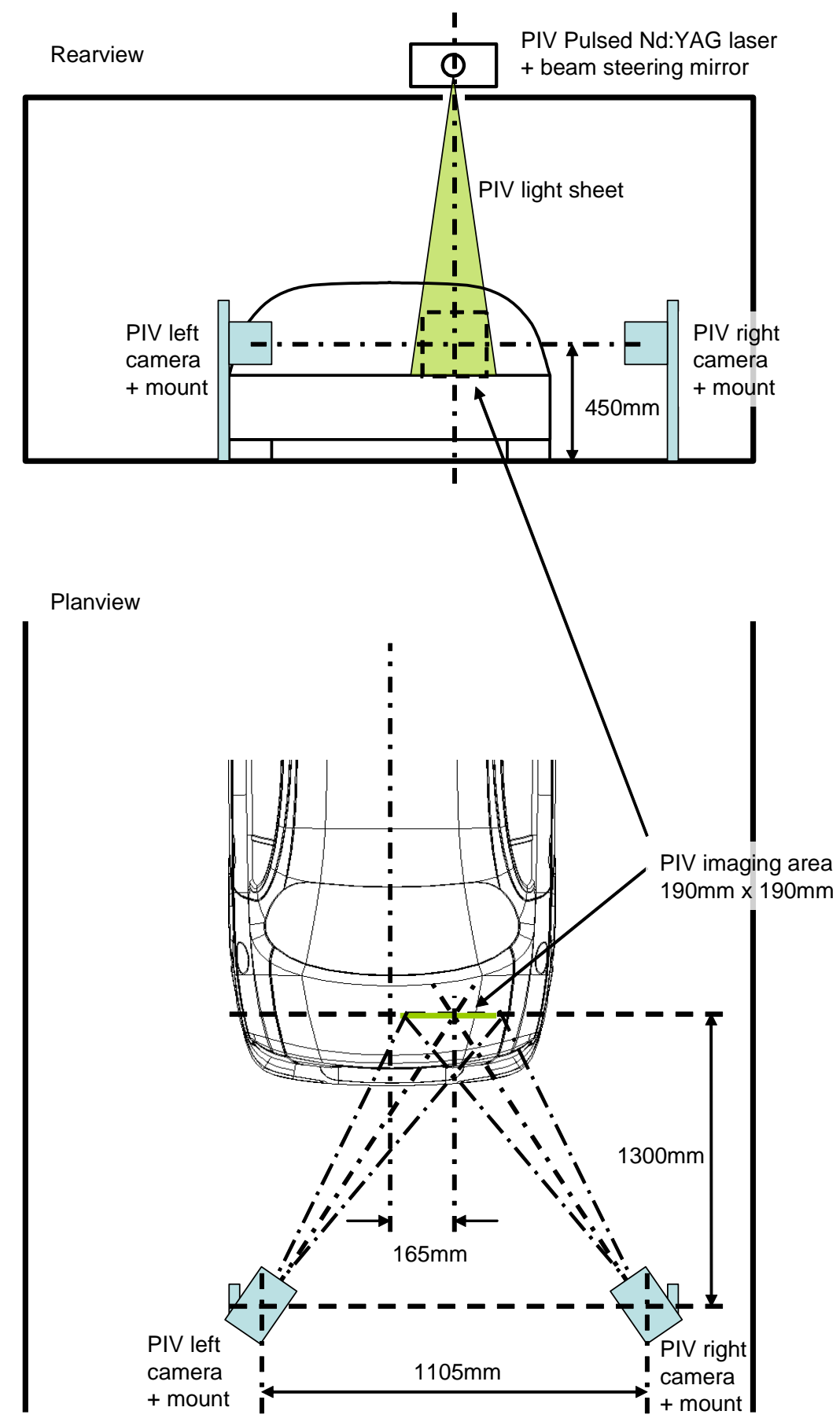

Figure 8 - Experimental arrangement for stereoscopic PIV measurements, top sketch rearview looking upwind, bottom sketch planview (flow from top to bottom) 


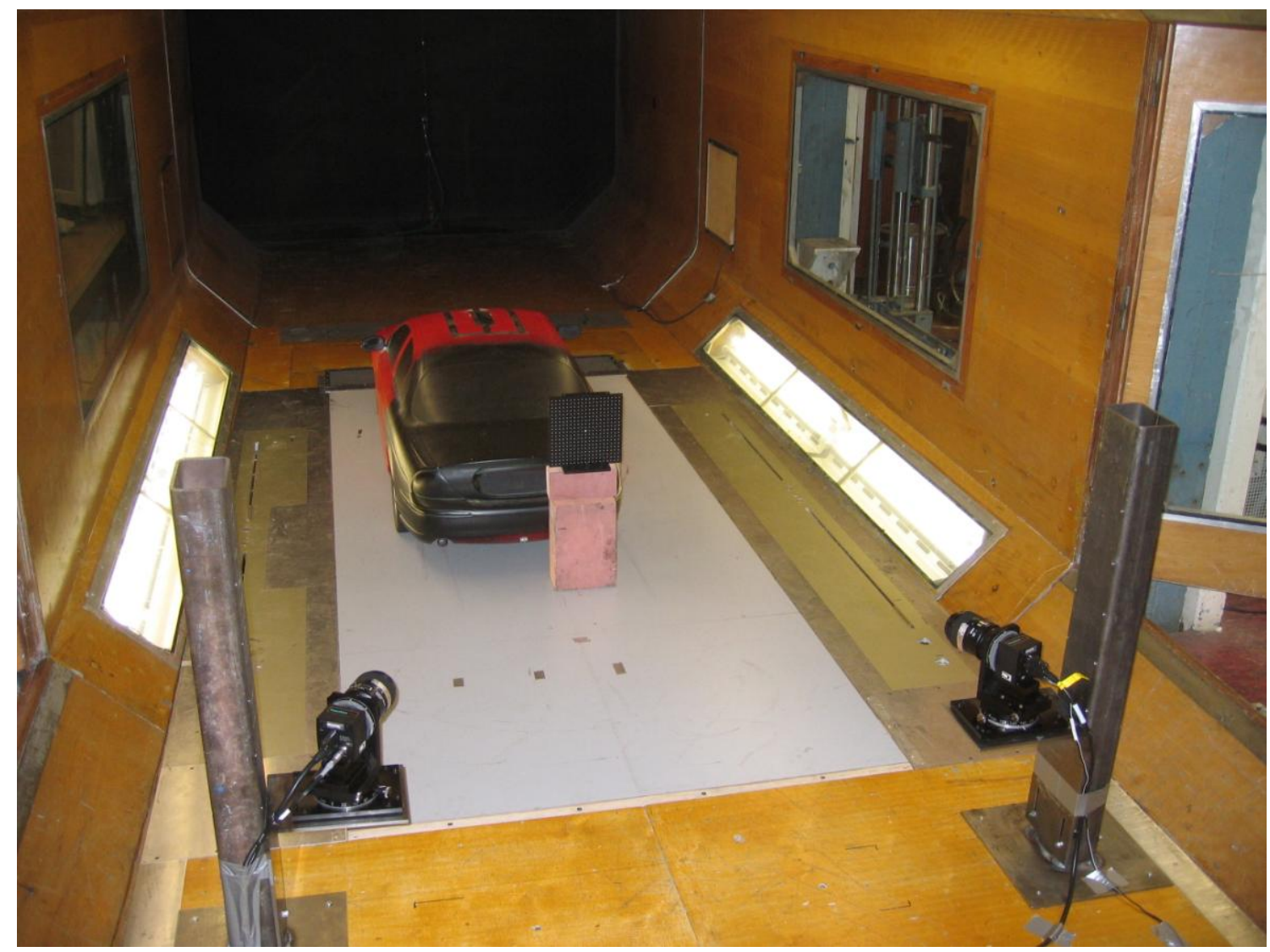

Figure 9 - View of stereoscopic PIV system with calibration grid 

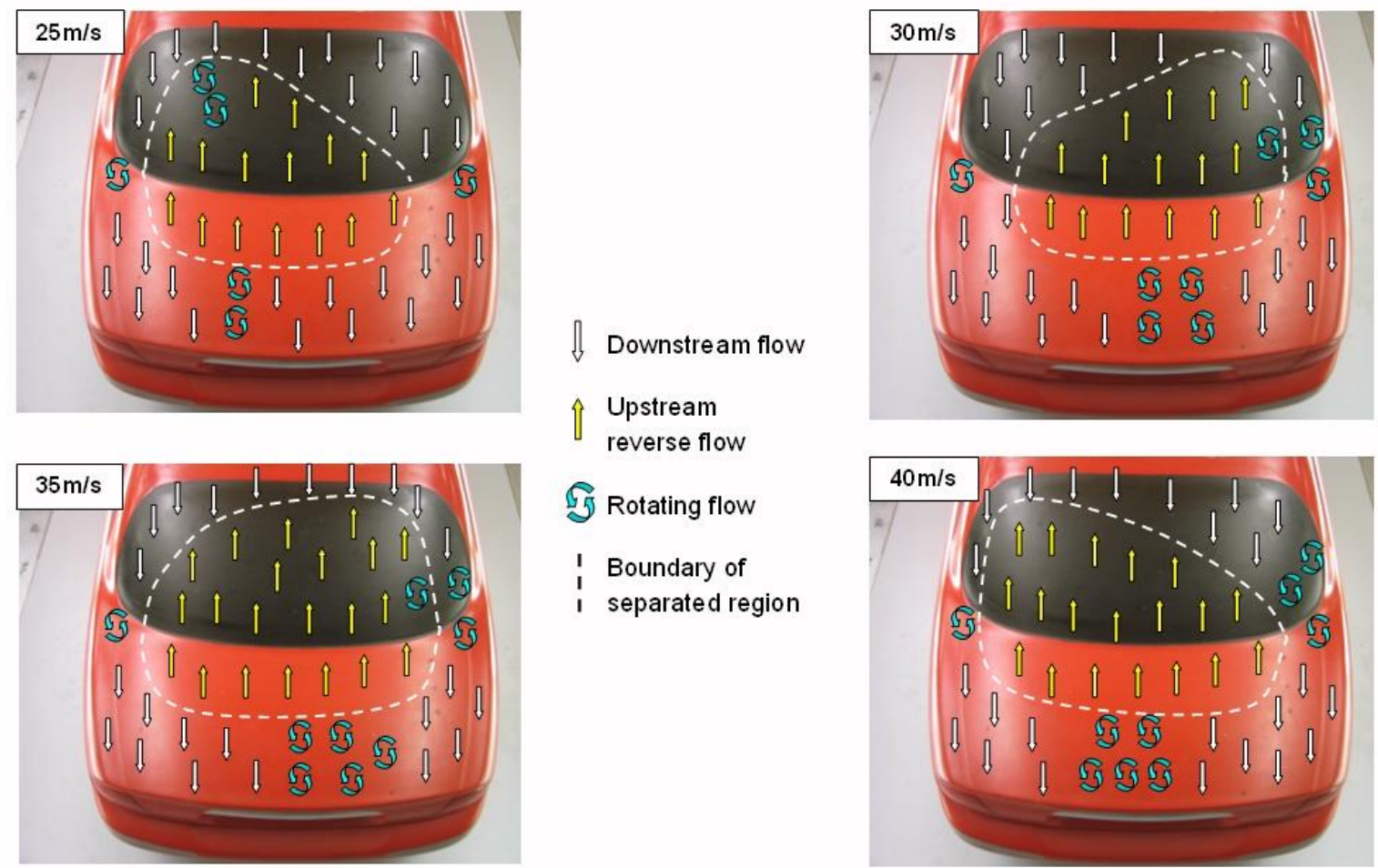

(5) Rotating flow

Boundary of

separated region

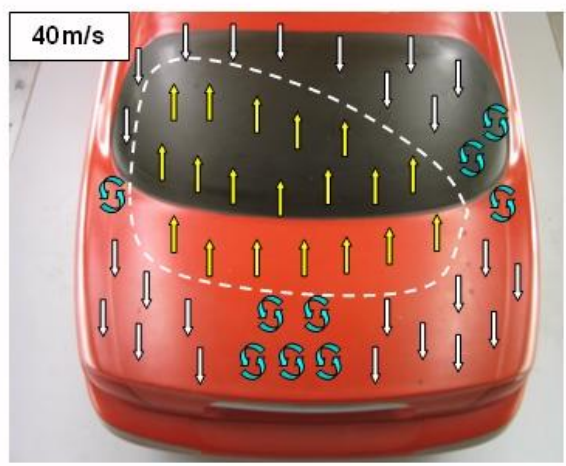

Figure 10 - Surface nylon tuft flow visualisation observations over model rearbody at different freestream speeds (flow from top to bottom in photographs) 


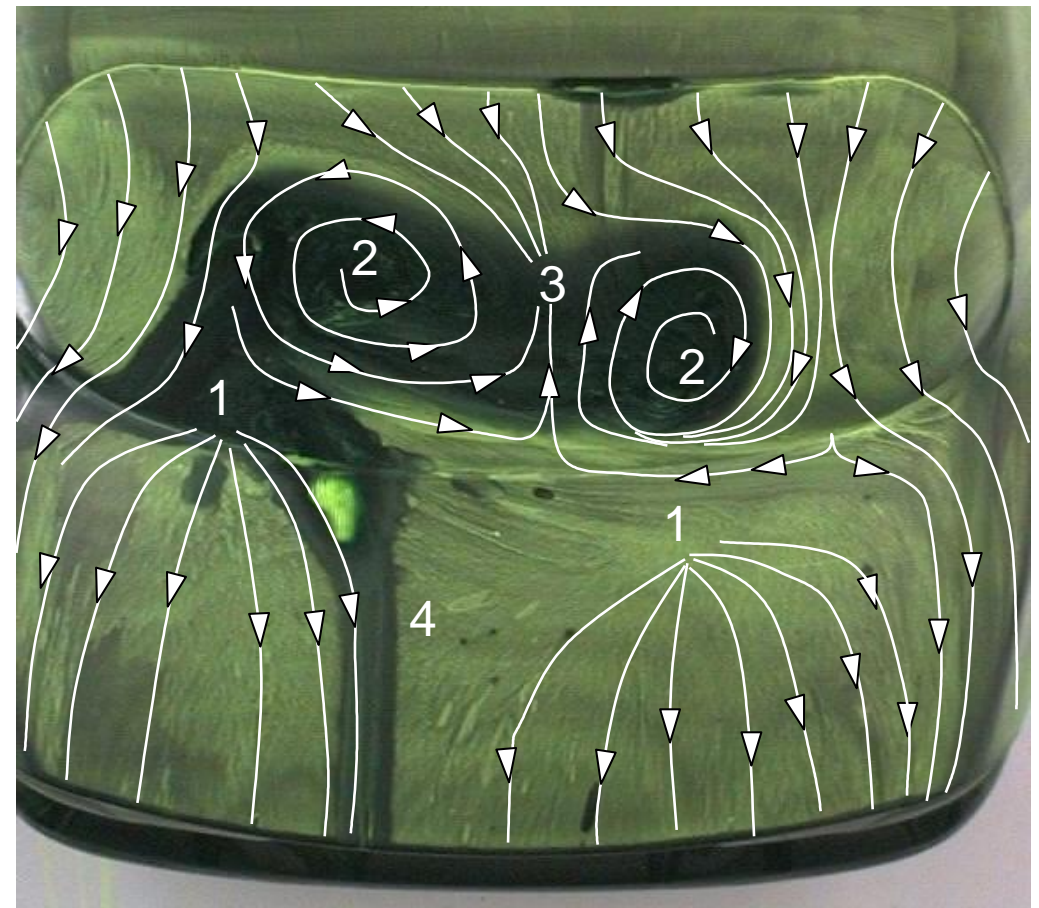

a)

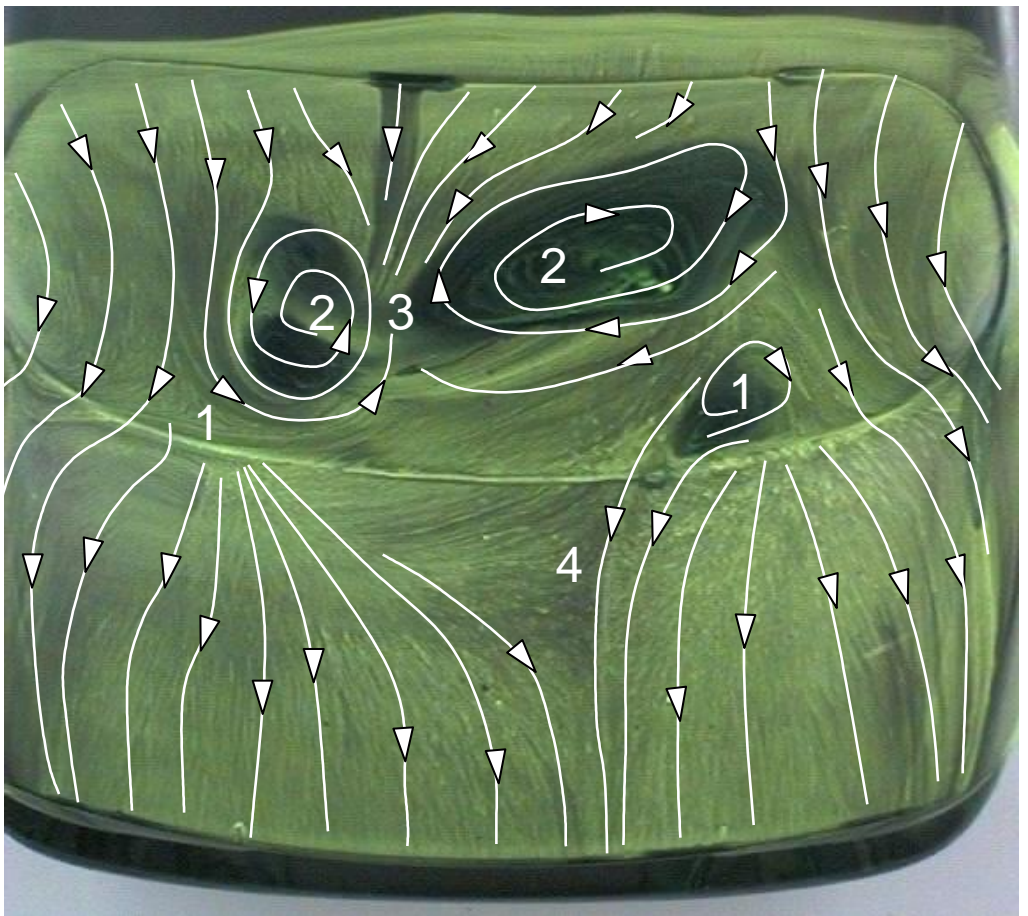

Figure 11 - Surface flow visualisation observations on model rearbody a) $25 \mathrm{~m} / \mathrm{s}(\mathrm{Re}=$ $\left.3.08 \times 10^{6}\right)$ b) $40 \mathrm{~m} / \mathrm{s}\left(\operatorname{Re}=4.93 \times 10^{6}\right)$ - freestream flow direction from top to bottom 

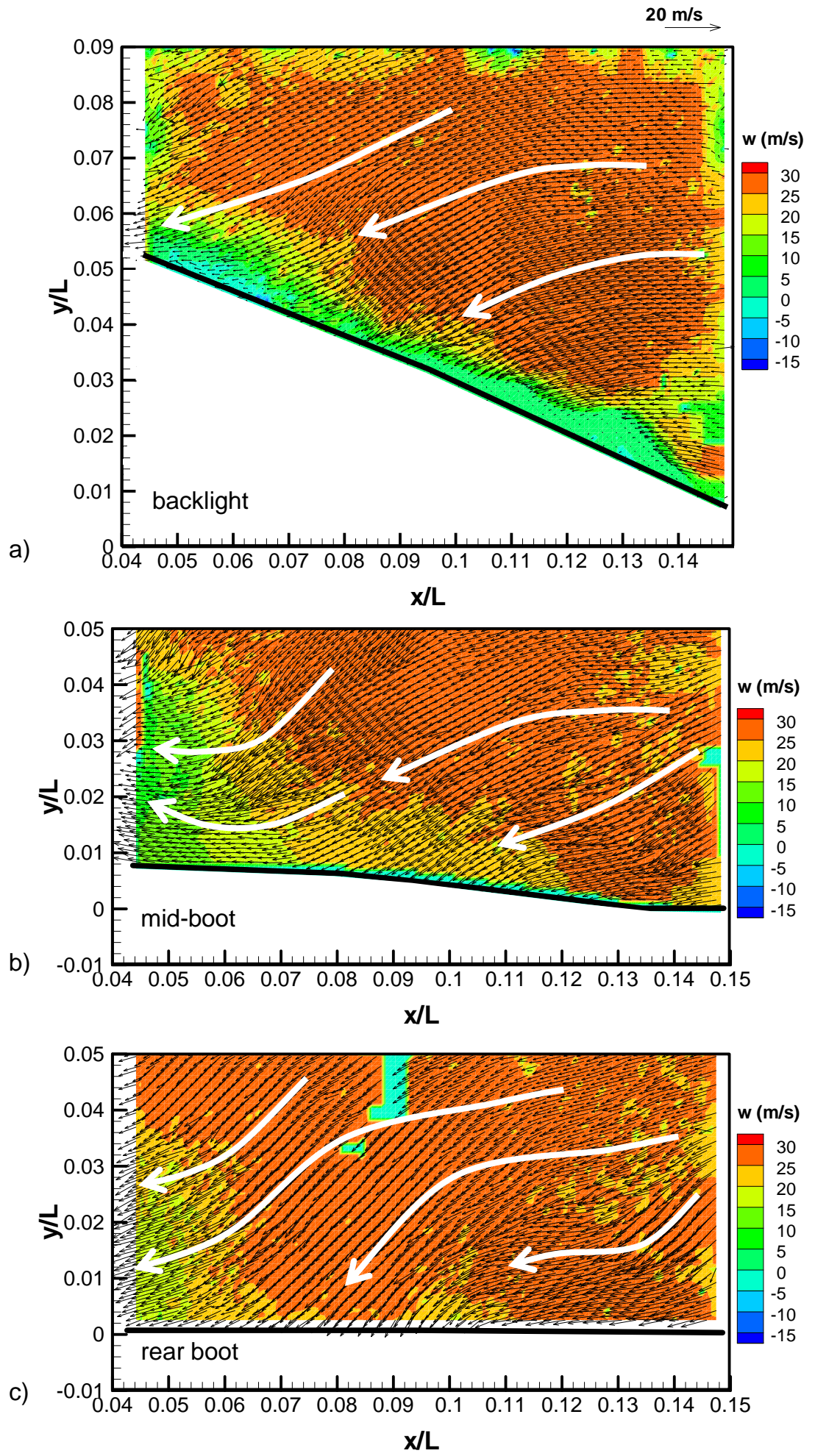

Figure 12 - 3D PIV data at $V_{\infty}=30 \mathrm{~m} / \mathrm{s}\left(\operatorname{Re}=3.69 \times 10^{6}\right)$ a) backlight b) mid-boot c) rear boot 


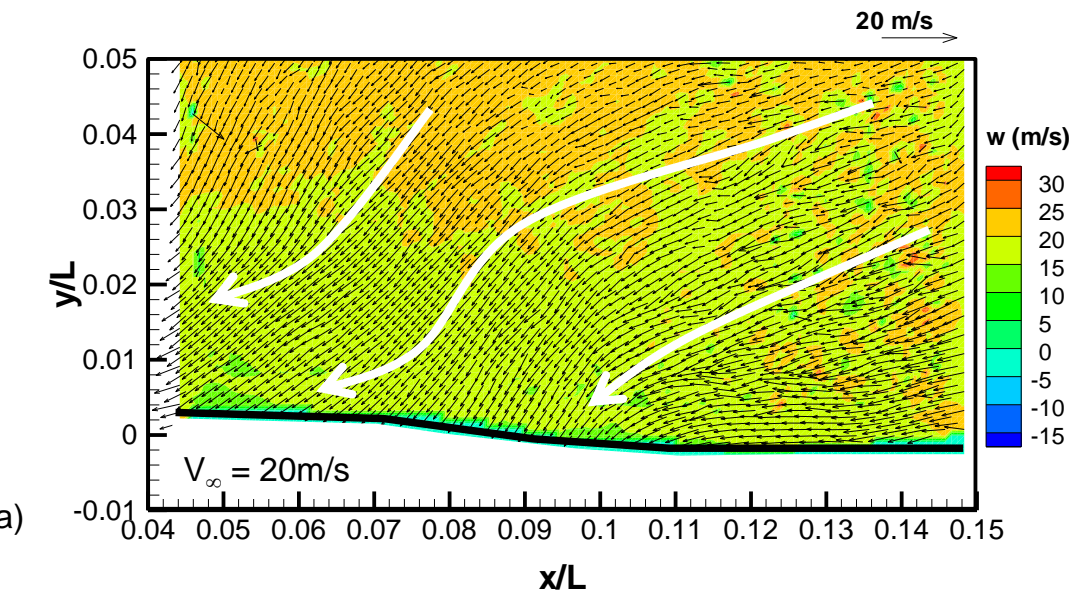

b)

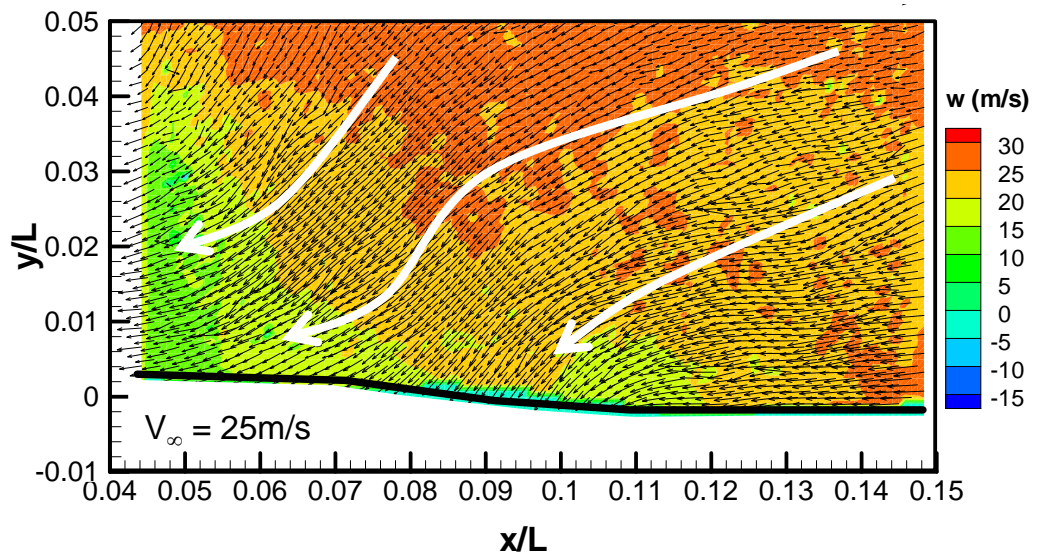

C)

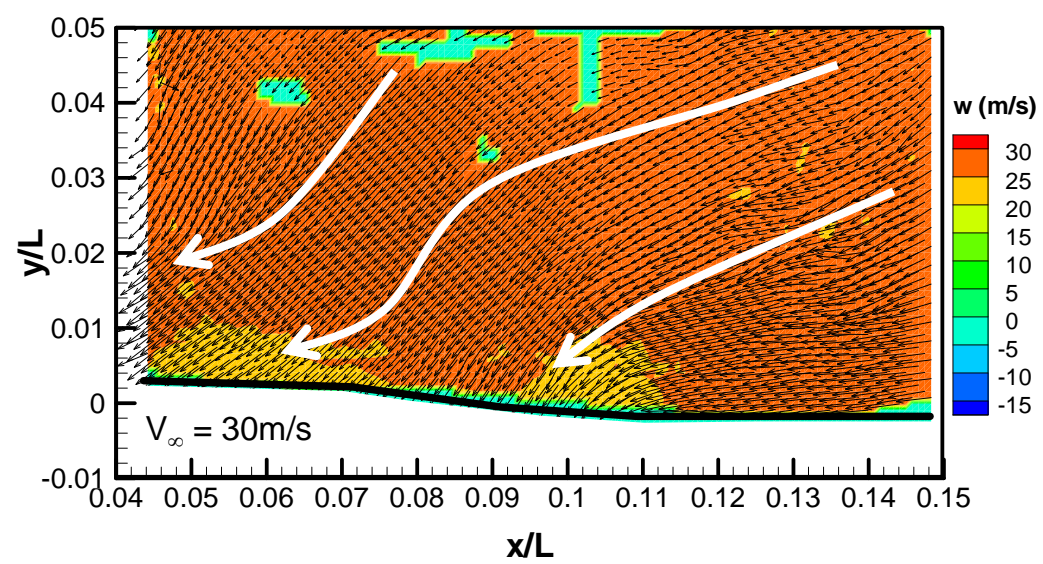

Figure 13 - 3D PIV Data at end-boot position a) $V_{\infty}=20 \mathrm{~m} / \mathrm{s}\left(R e=2.47 \times 10^{6}\right)$ b) $V_{\infty}=25 \mathrm{~m} / \mathrm{s}$ $\left(\operatorname{Re}=3.08 \times 10^{6}\right)$ b) $V_{\infty}=30 \mathrm{~m} / \mathrm{s}\left(\operatorname{Re}=3.69 \times 10^{6}\right)$ 

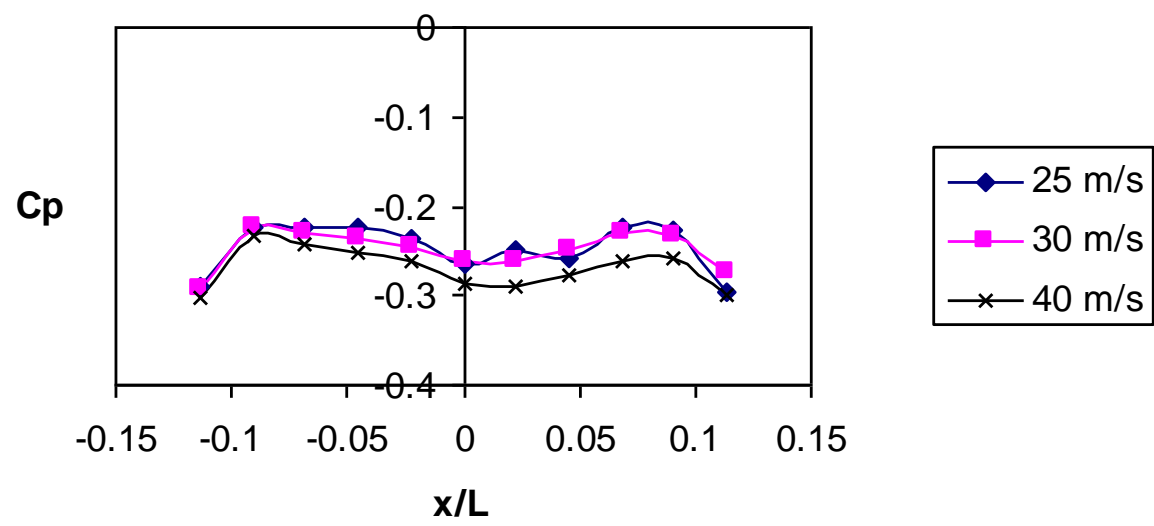

a)
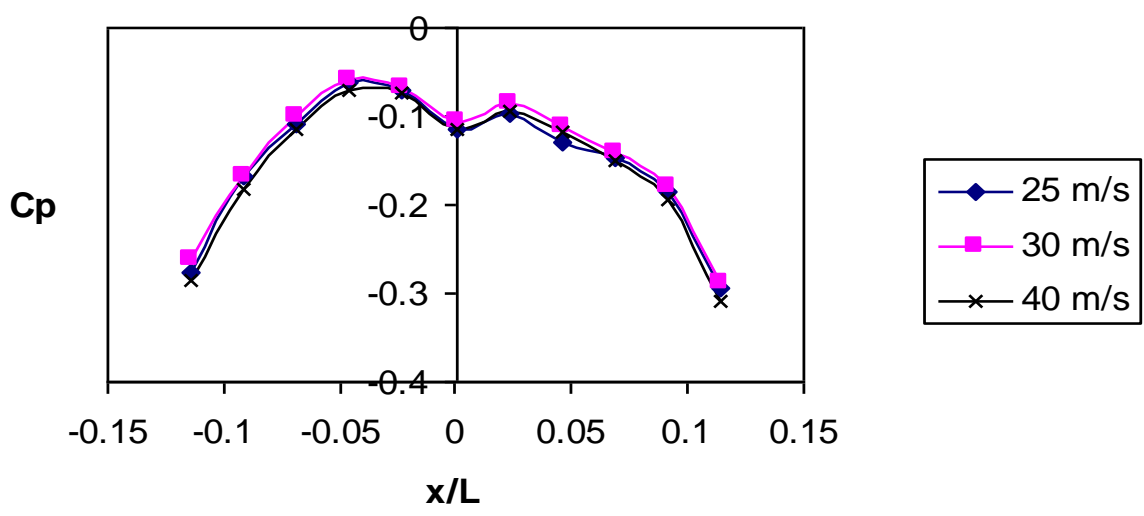

b)
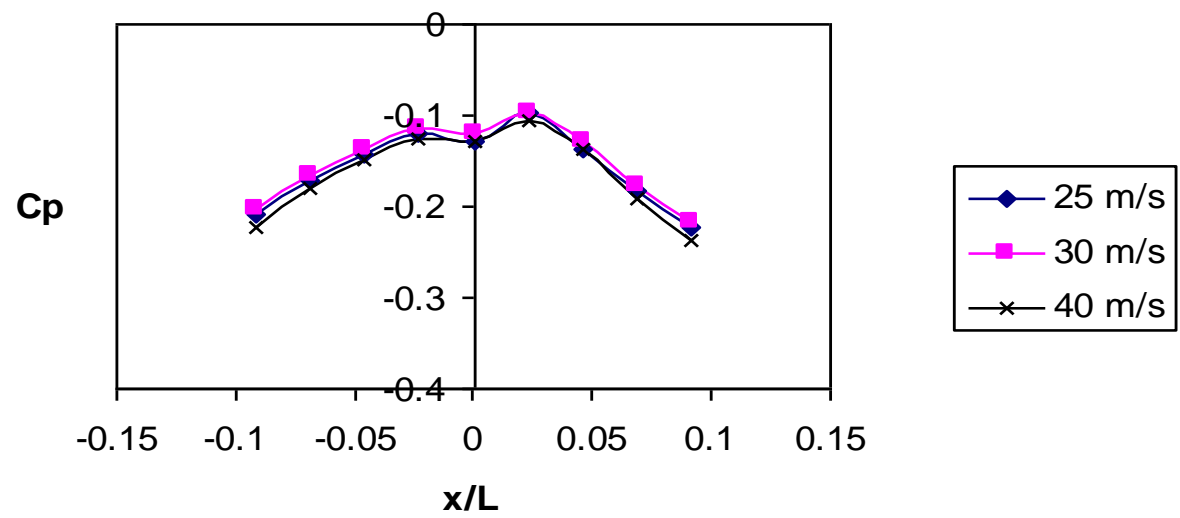

c)

Figure $14-a) c_{p}$ steady pressure distributions for the bootdeck region a) backlight region b) mid-boot region c) end-boot region 


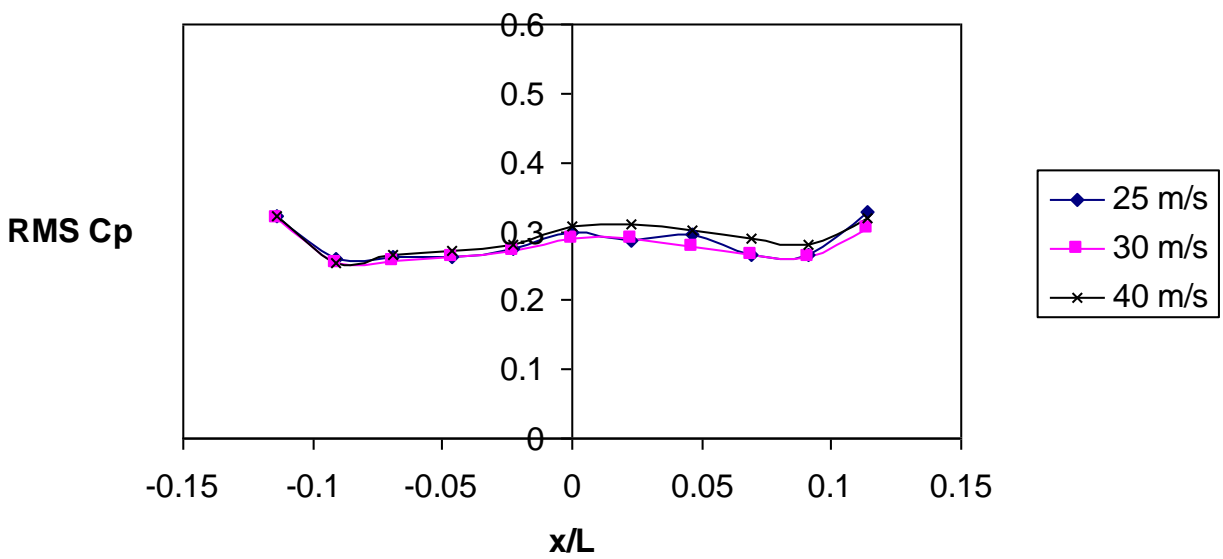

a)

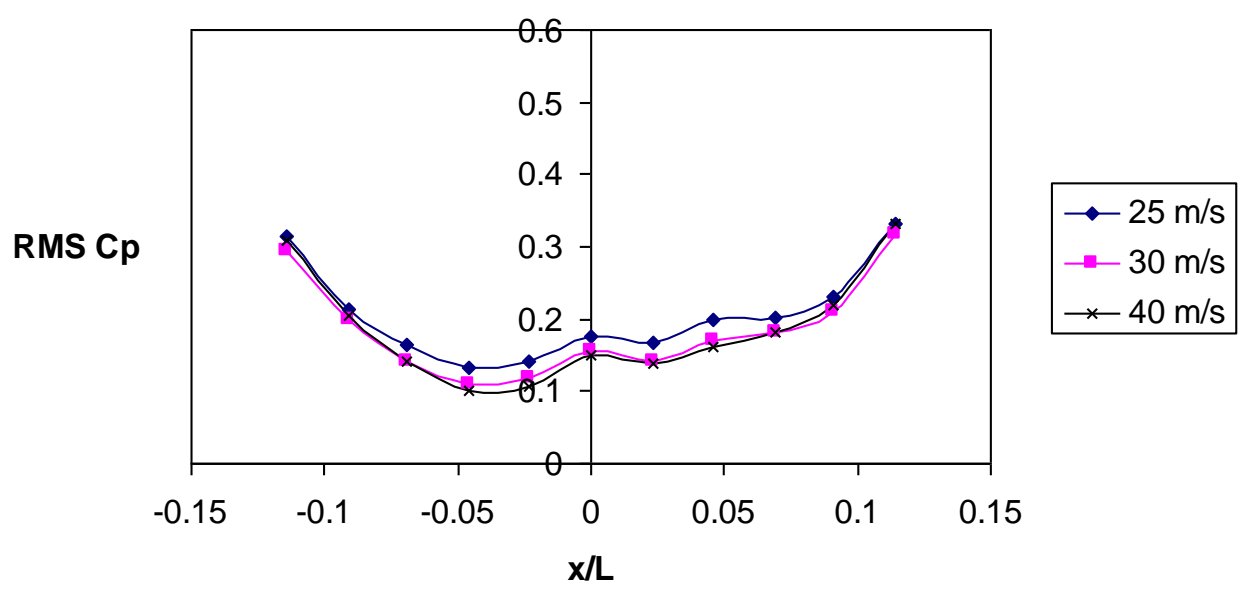

b)

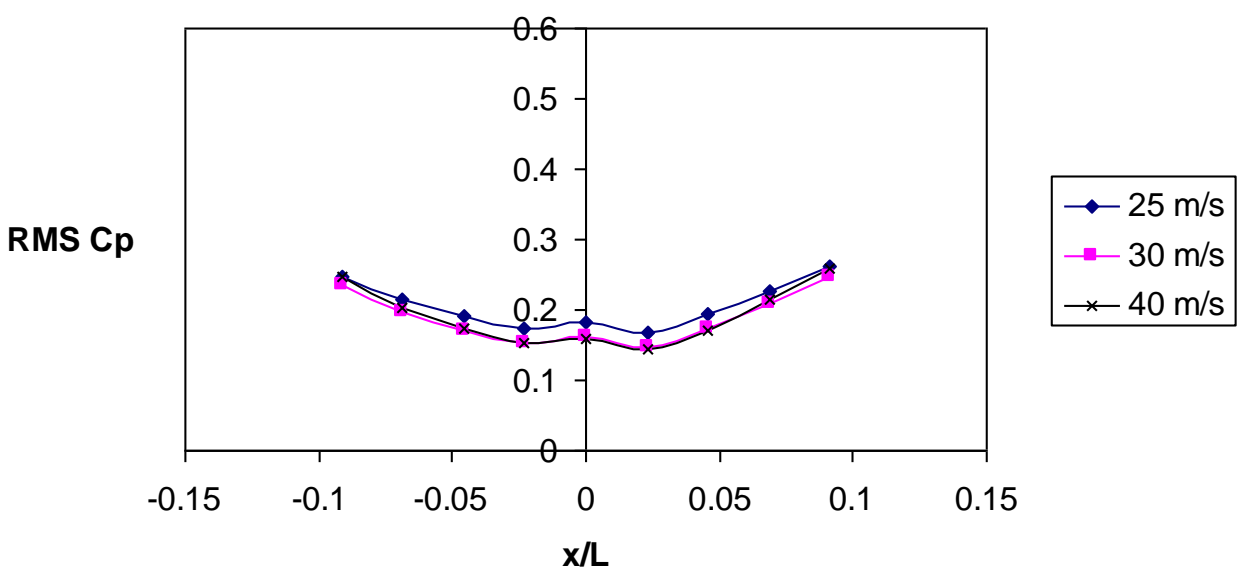

c)

Figure $15-a) c_{p}$ unsteady pressure distributions for the bootdeck region a) backlight region b) mid-boot region c) end-boot region 
Journal of Automotive Engineering

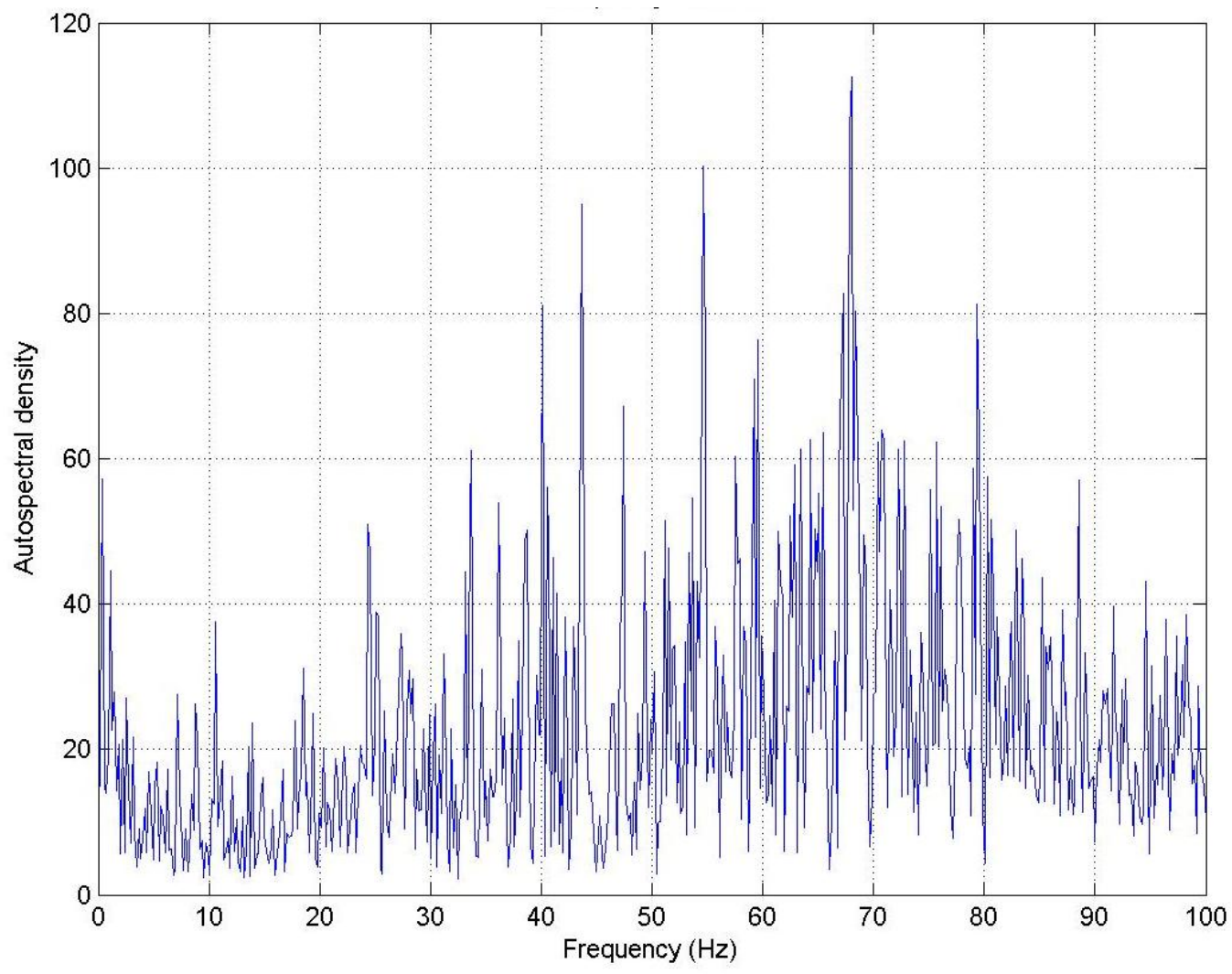

Figure 16 - spectral distribution from the end-boot region $\left(V_{\infty}=40 \mathrm{~m} / \mathrm{s}, \mathrm{Re}=4.93 \times 10^{6}, \mathrm{x} / \mathrm{L}=\right.$ 0) 